\title{
Mechanical Stress Protects Against Chondrocyte Pyroptosis through Lipoxin A4 via Synovial Macrophage M2 Subtype Polarization in an Osteoarthritis Model
}

\author{
Peng Shen \\ Shengjing Hospital of China Medical University \\ Shuangshuo Jia \\ Shengjing Hospital of China Medical University \\ Yang Wang \\ Shengjing Hospital of China Medical University \\ Xiaonan Zhou \\ Shengjing Hospital of China Medical University \\ Delong Zhang \\ Shengjing Hospital of China Medical University \\ Zhuangzhuang Jin \\ Shengjing Hospital of China Medical University

\section{Ziyuan Wang} \\ Shengjing Hospital of China Medical University

\section{Donghao Liu} \\ Shengjing Hospital of China Medical University

\section{Lunhao Bai} \\ Shengjing Hospital of China Medical University \\ Yue Yang ( $\nabla$ cmuyangyue@126.com )
}

Shengjing Hospital of China Medical University https://orcid.org/0000-0002-5712-3402

\section{Research Article}

Keywords: mechanical stimulation, lipoxin A4, synovial macrophages, osteoarthritis, pyroptosis

Posted Date: March 8th, 2022

DOI: https://doi.org/10.21203/rs.3.rs-1411791/v1

License: (a) This work is licensed under a Creative Commons Attribution 4.0 International License. Read Full License 


\section{Abstract}

Our previous study found that lipoxin $\mathrm{A}_{4}\left(\mathrm{LXA}_{4}\right)$ exerted therapeutic effects on osteoarthritis (OA). In this study, we evaluated the effects of $\mathrm{LXA}_{4}$ via synovial macrophage M1/M2 subtype polarization on chondrocyte pyroptosis and mechanisms during mechanical stimulation. Synovial macrophages were subjected to various $\mathrm{LXA}_{4}$ concentrations and cyclic tensile strain (CTS) conditions to determine optimal co-culture conditions. The effects of LXA 4 on chondrocyte pyroptosis, as represented by macrophage M1/M2 subtype polarization, were detected by western blot, immunofluorescence, and flow cytometry analyses. Forty male Sprague-Dawley rats were randomly divided into four groups ( $n=10)$ : control group (CG), OA group (OAG), OA with moderate-intensity treadmill exercise (OAE), and OAE + BOC-2 (an LXA 4 antagonist). All rats were evaluated using histology, enzyme-linked immunosorbent assay (ELISA), quantitative PCR, and western blot analyses. We found that $\mathrm{LXA}_{4}$ was downregulated in articular fluid and that CD 86/Arg 1 was up-regulated in the synovium of patients with increasing Kellgren-Lawrence grade. In vitro, CTS and LXA both promoted M2 subtype polarization of synovial macrophages, inhibiting the nuclear translocation of NF-kB p65 and the NLRP3 formation in chondrocytes. In vivo, the OAE treatment exerted protective effects on articular cartilage and facilitated M2 polarization of synovial macrophages. These effects were suppressed by BOC-2 treatment. We concluded that moderate CTS enhances the therapeutic effects of $\mathrm{LXA}_{4}$ by inhibiting the nuclear translocation of NF-kB p65 and NLRP3. Furthermore, the therapeutic effects of $\mathrm{LXA}_{4}$ during treadmill exercise in monoiodoacetate-induced $\mathrm{OA}$ were driven by promotion of synovial macrophage M2 subtype polarization.

\section{Introduction}

Osteoarthritis (OA) is a prevalent chronic joint disease characterized by structural alterations in the articular cartilage, subchondral bone, ligaments, capsules, synovial tissue, and periarticular muscles [1]; its main clinical features are pain and loss of function [2,3]. Globally, OA incidence is rising due to increasingly aged populations and obesity [4].

Acute inflammatory response is an immune mechanism that produces multiple mediators, including cytokines and chemokines [5, 6]. However, in the case of OA, overactive immune response or chronic inflammation can lead to tissue damage and fibrosis $[7,8]$. Lipoxin $\mathrm{A} 4\left(\mathrm{LXA}_{4}\right)$ is an eicosanoid acid produced in the capillaries and infrapatellar fat pad during cell-cell interactions, and is an end signal of inflammation $[9,10]$. In our previous studies, we found that LXA was rapidly produced and metabolized during exercise therapy for OA, suggesting an important role in the efficacy of this treatment $[11,12]$. This suggests that $L X A_{4}$ serves as an indicator for the curative effects of exercise, allowing for development of optimized therapeutic methods. Therefore, it is important to clarify how $L X A_{4}$ regulates inflammation during exercise therapy for OA.

Increasing evidence suggests that synovitis is related to OA progression [13]. Synovitis generates inflammatory mediators (TNF- $\alpha$ and IL-1 $\beta$ ), which are released into the synovial cavity and play a key role in OA pathogenesis and cartilage degeneration. However, the cellular and molecular processes that occur in the OA synovium are poorly understood, and further research is required to develop novel therapeutics [14]. The synovium of the knee joint is divided into two layers: the intima and the subintima. The intima comprises synovial macrophages and fibroblasts, which are important to the knee joint microenvironment and affect the joint through the interaction of articular chondrocytes $[15,16]$. Chondrocytes, which comprise articular cartilage, synthesize extracellular matrix and depend on intracellular signals generated in response to the microenvironment $[8,17]$. Synovial macrophages are tissue-resident immune cells with critical roles in maintaining homeostasis and fighting inflammation. Locally-renewed and protective tight junction-mediated macrophage barrier functions are important for our general understanding of macrophage roles in health and disease [18]. Macrophages exert strong plasticity in response to a variety of microenvironmental 
stimuli, and can polarize into either of two functional states to generate a spectrum of functional phenotypes. Classical, or M1, macrophage polarization executes kill functions, whereas alternative, or M2, polarization is responsible for repair [19-21]. Recent studies have found that macrophage polarization is closely related to OA pathogenesis [22], and is regulated by a variety of signal pathways. Pyroptosis is a pro-inflammatory type of programmed cell death caused by inflammation, and includes the activation of Nod-like receptor protein 3 (NLRP3)/ caspase-1. This process induces cell rupture and release of contents in a manner similar to that of necrosis, but is caspase-driven, more like apoptosis [23, 24]

Appropriate exercise regimens are critical aspects of OA treatment plans [25-27]. Bodily tissues and cells are continuously exposed to complex mechanical cues from the environment. Mechanical stimulation affects cell proliferation, differentiation, and migration, as well as determining tissue homeostasis and repair [28]. Although synovial macrophages experience mechanical stress during exercise therapy, little is known about how these mechanical processes regulate immune responses.

Here, we show that cyclic tensile stress (CTS), similar to that experienced in the joint, initiates an inflammatory response via synovial macrophage polarization. The mechano-transduction signaling pathways of synovial macrophages are complex, and the mechanisms by which they convert mechanical stimuli into biochemical signals requires further investigation.

Several studies have emphasized the importance of exercise therapy to OA. However, most have focused on single cells, such as chondrocytes, synovial fibroblasts, or bone cells $[29,30]$. Little is known regarding the complexity of how this treatment functions in living organisms. Thus, the effects of mechanical stimulation on chondrocyte behavior in multi-population systems is unclear.

In our preliminary experiment of this study, M2 polarization markers were upregulated, and $\mathrm{LXA}_{4}$ was down-regulated in patients with high Kellgren-Lawrence grades. This finding was explored through in vivo and in vitro experiments. In vitro, the indirect effects of synovial macrophages on chondrocytes were studied by establishing a co-culture system using transwell chambers. Synovial macrophages were subjected to variable LXA 4 and CTS conditions. M2 polarization was found to inhibit the nuclear translocation of NF-KB p65 and the formation of NLRP3 in chondrocytes. In vivo, we used $B O C-2$, an antagonist of the $L X A_{4}$ receptor, to evaluate the effects of $L X A_{4}$ on synovial macrophage M2 polarization during moderate-intensity treadmill exercise in a rat model of monoiodoacetate (MIA)-induced OA [29]. These data provide new insights into the role of synovial macrophages in OA pathogenesis, and suggest that LXA is suitable for assessing the effects of treadmill exercise on OA treatment and progression. Our findings offer to improve the development of exercise programs for patients with OA.

\section{Materials And Methods}

\subsection{Human OA synovium, articular fluid, and clinicopathological features}

This study was approved by the ethics committee of the Shengjing Hospital of China Medical University (No. 2017PS237K). All patients consented to their inclusion in this study. The synovium, articular fluid, and clinicopathological features of patients with different Kellgren-Lawrence grades were collected from January 2019 to August 2021, based on data from 184 patients treated by arthroscope in the Fourth Department of Orthopedic Surgery in our hospital. The inclusion criteria were: 1) epiphyseal closure, 2) sign informed consent, 3) no definite history of trauma within three months, and 4) no drug therapy within three months. The exclusion criteria were: 1) tumor and/or 
infection of knee tissue, 2) lack of obtained tissue samples, or 3) lack of imaging examination. (Table 1, Fig. 1A). Cartilage specimens from the tibial plateau, as well as medial and lateral femur condyles, removed after total knee arthroplasty were selected. The synovial tissue around the diseased cartilage was also collected.

Table 1

Baseline characteristics of patients

\begin{tabular}{|c|c|c|c|c|c|c|c|c|}
\hline $\begin{array}{l}\text { Basic } \\
\text { information }\end{array}$ & & $\begin{array}{l}\text { Kellgren- } \\
\text { Lawrence } \\
\text { grade } 0\end{array}$ & $\begin{array}{l}\text { Kellgren- } \\
\text { Lawrence } \\
\text { grade } 1\end{array}$ & $\begin{array}{l}\text { Kellgren- } \\
\text { Lawrence } \\
\text { grade } 2\end{array}$ & $\begin{array}{l}\text { Kellgren- } \\
\text { Lawrence } \\
\text { grade } 3\end{array}$ & $\begin{array}{l}\text { Kellgren- } \\
\text { Lawrence } \\
\text { grade } 4\end{array}$ & Statistic & $\begin{array}{l}\mathrm{P} \\
\text { value }\end{array}$ \\
\hline \multirow[t]{2}{*}{$\begin{array}{l}\text { Biological } \\
\text { Sex }\end{array}$} & Male & 13 & 15 & 12 & 15 & 12 & $\begin{array}{l}x^{2}= \\
5.501\end{array}$ & 0.240 \\
\hline & Female & 15 & 18 & 19 & 28 & 37 & & \\
\hline \multirow[t]{2}{*}{ Age (years) } & $\otimes 65$ & 28 & 33 & 16 & 8 & 5 & $\begin{array}{l}x^{2}= \\
108.99\end{array}$ & $\begin{array}{l}< \\
0.001\end{array}$ \\
\hline & $\geq 65$ & 0 & 0 & 15 & 35 & 44 & & \\
\hline \multirow[t]{4}{*}{$\begin{array}{l}\text { Obesity } \\
\text { gradation }\end{array}$} & $\begin{array}{l}\text { Under } \\
\text { weight }^{a}\end{array}$ & 2 & 1 & 0 & 1 & 2 & $\begin{array}{l}x^{2}= \\
52.113\end{array}$ & $\begin{array}{l}< \\
0.001\end{array}$ \\
\hline & $\begin{array}{l}\text { Normal } \\
\text { weight }\end{array}$ & 22 & 27 & 21 & 24 & 10 & & \\
\hline & Overweight & 4 & 4 & 8 & 15 & 23 & & \\
\hline & Obesity & 0 & 1 & 2 & 3 & 14 & & \\
\hline
\end{tabular}

\subsection{Western blotting}

Synovial tissue was washed twice in ice-cold potassium-buffered saline (PBS). Cytoplasm protein levels were measured using a bicinchoninic acid assay kit (Beyotime, Shanghai,China) according to manufacturer instructions. Equal protein concentrations $(40 \mu \mathrm{g})$ were separated by SDS-PAGE and transferred to polyvinylidene difluoride membranes.

Membranes were blocked with $1 \%$ bovine serum albumin (BSA) in tris-buffered saline (TBS) containing $0.1 \%$ Tween-20 (TBST) at room temperature for $2 \mathrm{~h}$. They were then incubated overnight at $4^{\circ} \mathrm{C}$ with the following primary antibodies: rabbit monoclonal anti-vascular endothelial growth factor (VEGF) receptor 1 (ab32152, 1:1000; Abcam; molecular weight $151 \mathrm{kDa}$ ); rabbit monoclonal anti-NLRP3 (ab210491, 1:1,000; Abcam; 118 kDa); rabbit monoclonal anti-MMP13 (ab51072, 1:1000; Abcam; 54 kDa); rabbit monoclonal anti-caspase-1 (ab108362, 1:5,000; Abcam; 45 kDa); rabbit monoclonal anti-pro caspase-1 (ab179515, 1:1,000; Abcam; 45 kDa, 42 kDa); rabbit monoclonal anti- TGF $\beta 1$ (ab215715, 1:1000; Abcam 44 kDa); mouse monoclonal anti- $\beta$-actin (60008-1-lg, 1:5,000, Proteintech Group; 42 kDa); rabbit polyclonal anti-CD86 (ab220188, 1 g g/mL, Abcam; $38 \mathrm{kDa}$ ); rabbit polyclonal anti-Arg 1 (16001-1-AP, 1:20000; Proteintech 36 kDa); rabbit monoclonal anti-pro IL-1 $\beta$ antibody (ab216995, 1:1000; Abcam; 29 kDa, 35 kDa); rabbit monoclonal anti-IL-10 (ab133575, 1:1000; Abcam; 18 kDa). After washing three times with TBST, membranes were incubated with IgG-horseradish peroxidase-conjugated secondary antibodies (1:10000, Canlife) at room temperature for $2 \mathrm{~h}$. After washing in TBST immunoreactivity was detected using enhanced chemiluminescence and proteins were quantified using Quantity ONE (Bio-Rad, Hercules, CA, USA) software. $\beta$-actin was used as an internal control. 


\subsection{Enzyme-linked immunoassay (ELISA) of LXA 4 , tumor necrosis factor- $a($ TNF- $a)$, and IL-1 $\beta$}

$\mathrm{LXA}_{4}$ in articular fluid of patients was analyzed using ELISA kits (Shanghai Kemin Biotechnology Co. Ltd., Shanghai, China) according to manufacturer instructions. TNF- $\alpha$ and IL-1 $\beta$ levels in the knee intra-articular lavage fluid (IALF) and serum were also determined using ELISA kits (Tongwei) according to manufacturer instructions. Protein levels in IALF were measured to ensure that the dilution ratio was equal.

\subsection{Isolation and culture of synovial macrophages and chondrocytes from patients with OA}

Synovium and articular cartilage at the site of the lesion were collected from two males and four females who underwent total knee replacement, aged 58-71 years. Synovial macrophages and chondrocytes were derived from the synovium and articular cartilage of knee joints. Macrophages and chondrocytes we isolated from patients with a Kellgren-Lawrence grade of 4, ensuring consistent cellular responses to CTS and/or LXA 431,32$]$. Tissues were collected into sterile PBS.

Fat and connective tissue were removed and tissue was digested in $1 \mathrm{mg} / \mathrm{mL}$ collagenase (Sigma Aldrich, St. Louis, $\mathrm{MO}, \mathrm{USA}$ ) for $2-3 \mathrm{~h}$ at $37^{\circ} \mathrm{C}$. Cells were separated from undigested tissue using a $70 \mu \mathrm{m}$ cell strainer (BD, Franklin Lakes, NJ, USA) and cultured in $25 \mathrm{~cm}^{2}$ cell culture flasks in Dulbecco's Modified Eagle Medium (DMEM) (Gibco BRL, Grand Island, NY, USA) with 10\% fetal bovine serum (Gibco BRL) and antibiotics (100 U/mL penicillin, $100 \mu \mathrm{g} / \mathrm{mL}$ streptomycin, and $0.1 \mathrm{mg} / \mathrm{mL}$ fungizone) in a humid $5 \% \mathrm{CO}_{2}$ atmosphere at $37^{\circ} \mathrm{C}$. The cells were transferred into 25 $\mathrm{cm}^{2}$ culture flasks at a density of $5 \times 10^{6}$ cells/flask. At approximately 80-90\% confluence, cells were detached using $0.25 \%$ trypsin and split into a 1:3 ratio. For all experiments, first and second cell passages were used. Synovial macrophages were identified by immunofluorescence staining with anti-CD 14 antibody (ab183322, 1:100; Abcam) (Fig. 2A, left).

Articular cartilage sections were sequentially digested in pronase $(2 \mathrm{mg} / \mathrm{mL})$ and collagenase $\mathrm{D}(1 \mathrm{mg} / \mathrm{mL} ;$ Roche, Basel, Switzerland). Cells were cultured as previously described [33] and identified by immunohistochemical staining with anti-COLII antibody (ab34712, 1:100; Abcam) (Fig. 2A, right).

\subsection{Exposure of synovial macrophages to CTS and LXA 4}

Synovial macrophages were grown on fibronectin-coated Bioflex six-well culture plates (Flexcell International, Hillsborough, NC, USA) to 80-90\% confluence. CTS experiments were conducted using the FX-5000 Flexcell system (Flexcell International, McKeesport, PA, USA). To provide uniform radial and circumferential strain on the membrane, plates were placed in a loading station (in a $5 \% \mathrm{CO}_{2}$ incubator), such that when a vacuum was applied, the membrane deformed across the post face, creating a uniform bi-axial strain. Synovial macrophages were subjected to CTS (10\%, $0.5 \mathrm{~Hz})$ of different durations $(0,6,12,24,48,72$, or $96 \mathrm{~h})$, and $\mathrm{LXA}_{4}$ concentrations $(0,0.5,1,2$, or $4 \mathrm{nM})$ for $24 \mathrm{~h}$, following the methods of our previous study [11]. After these steps, we selected the optimal conditions for further study (Fig. 2B).

\subsection{Determination of reactive oxygen species (ROS)}

Synovial macrophages were exposed to CTS $(10 \%, 0.5 \mathrm{~Hz}, 24 \mathrm{~h})$ or LXA $4(1 \mathrm{nM})$, and chondrocytes were harvested at 24 h. ROS production was measured using 2',7'-dichlorodihydrofluorescein diacetate (DCFH-DA) (S0033, Beyotime), which is directly oxidized by ROS (e.g. superoxide ion, hydrogen peroxide, hydroxyl ions). Chondrocytes were incubated with 
$10 \mu \mathrm{M}$ DCFH-DA for $45 \mathrm{~min}$ at $37^{\circ} \mathrm{C}$ in the dark. After this, they were washed three times in PBS. Fluorescence was detected by fluorescent microscopy and measured using the BD FACSCalibur instrument (BD Bioscience; $488 \mathrm{~nm}$ excitation, $525 \mathrm{~nm}$ emission).

\subsection{Co-culture}

Synovial macrophages were placed in the bottom compartment of a transwell system, and chondrocytes placed in the top. Cells were cultured with $0.4 \mu \mathrm{m}$ inserts. Both cell types were mixed at a ratio of 3:1. Growth conditions were selected as previously described. Cell viability was $>95 \%$ before study commencement. Experiments were repeated at least three times with cells from different donors (one patient per experimental trial). Appropriate LXA concentrations $_{4}$ $(1 \mathrm{nM})$ and CTS conditions ( $10 \%, 0.5 \mathrm{~Hz}, 24 \mathrm{~h}$ ) were applied to synovial macrophages. After $24 \mathrm{~h}$ incubation, chondrocytes were collected for further study.

\subsection{Immunofluorescence of chondrocytes}

After washing in PBS and fixing in 4\% paraformaldehyde for 20 min at room temperature, chondrocytes were permeabilized with $0.5 \%$ Triton $\mathrm{X}-100$ for 30 min and incubated with blocking solution (5\% BSA) for 30 min at room temperature. Rabbit polyclonal anti-NF-KB p65 antibody (AB21014, 1:50; Abcam) was then added, and cells were incubated overnight at $4^{\circ} \mathrm{C}$. Cells were then stained with Alexa Fluor ${ }^{\circledR} 488$ conjugated anti-rabbit antibody for 60 min at room temperature in the dark. Cytoskeleton structures were stained with phalloidin for $60 \mathrm{~min}$ at $37^{\circ} \mathrm{C}$. Nuclei were counterstained with 4, 6-diamidino-2-phenylindole (DAPI) for 2 min. After washing, cells adhering to Bioflex membranes were mounted in PBS with $20 \%$ glycerol. Chondrocytes were observed under a confocal microscope (Olympus, Tokyo, Japan).

\subsection{Transmission electron microscopy (TEM)}

After washing with PBS, the chondrocytes were harvested and centrifuged for 2 min at 2500 rpm before being fixed in cold $2.5 \%$ glutaraldehyde for $30 \mathrm{~min}$. Samples were fixed again in $1 \% \mathrm{OsO}_{4}$ for $1 \mathrm{~h}$, dehydrated, and embedded in epoxy resin. Ultrathin tissue sections $(60 \mathrm{~nm})$ were stained with uranyl acetate and lead citrate. The sections were examined via TEM (Hitachi 800, Tokyo, Japan) to observe cellular morphology.

\subsection{Study animals}

This study was approved by the ethics committee of Shengjing Hospital of China Medical University (No. 2017PS237K). Forty male Sprague-Dawley rats (230 g $\pm 10 \mathrm{~g}$; specific pathogen-free) were obtained from HFK Bioscience Co. Ltd. (Beijing, China). Rat maintenance and care followed committee guidelines as previously described $[12,29]$. Rats were adapted to laboratory conditions for one week prior to experimental procedures. All rats were familiarized with the ZH-PT treadmill exercise machine (Zhongshidichuang Science \& Technology Development Co. Ltd., Beijing, China) for one week at $10 \mathrm{~m} / \mathrm{min}$ for $10 \mathrm{~min} /$ day to reduce stress. All rats successfully adapted to the treadmill exercise [13]

\subsection{OA model and treadmill protocols}

After adaption to treadmill exercises, rats were randomly divided into four study groups $(n=10)$. Prior to treatment, all rats were anesthetized with $1.5 \%$ pentobarbital sodium $(0.2 \mathrm{~mL} / 100 \mathrm{~g}$, intraperitoneal injection). The treatments were as follows: 1) Control group (CG), intra-articular injection of $50 \mu \mathrm{L}$ sterile saline per cavity; 2) OA group (OAG), knee joint inflammation induced by intra-articular injection of MIA (1 mg per cavity in $50 \mu \mathrm{L}$ sterile saline) through the lateral part of the inferior patellar ligament and into the bilateral knee joint cavity; 3 ) OAE, OAG conditions with moderate treadmill exercise $\left(19.3 \mathrm{~m} / \mathrm{min}\right.$ with a $5^{\circ}$ inclination, $60 \mathrm{~min} /$ day, $5 \mathrm{~d} /$ week for four weeks with appropriate photo-, acoustic-, and electric stimulation); 4) OAE + BOC-2, OAE conditions plus BOC-2 (an antagonist of LXA ; Absin, Shanghai, China). BOC- 
$2(50 \mu \mathrm{g} / \mathrm{kg})$ was administered by intraperitoneal injection before every treadmill exercise (10) (Fig. 5A). Because we demonstrated in previous studies that moderate exercise intensity showed an optimal therapeutic effect on osteoarthritis, we did not include low- or high-intensity exercise in this study [11,34].

\subsection{Sampling, tissue preparation, and histology}

All rats were euthanized by cervical dislocation. The left knee joints were dissected and fixed in $4 \%$ paraformaldehyde solution. Skin and muscles along the patellar ligament were removed to open the capsula articularis, with care to avoid damage to the cartilage and synovium. Next, using a scalpel or rongeur, articular cartilage specimens were removed from the weight-bearing areas of the right femur and tibia condyles to analyze through western blot and quantitative PCR (qPCR). Synovium specimens were also removed. All tissue was stored at $-80^{\circ} \mathrm{C}$. Left knee joint tissue samples were stored in $4 \%$ paraformaldehyde for $7 \mathrm{~d}$, then washed in water for $5 \mathrm{~h}$ and transferred to a $20 \%$ EDTA solution (Jianglai Reagent Co., Ltd, Shanghai, China) to decalcify for $21 \mathrm{~d}$. The solution was changed every $3 \mathrm{~d}$. Decalcified samples were dehydrated in an ethanol gradient series and embedded in paraffin. Serial $5 \mu \mathrm{m}$ sagittal sections were removed from tibiofemoral joints for histological examination. Sections were stained in hematoxylin \& eosin (HE) and toluidine blue to observe cartilage. Next, sections were observed with a ScanScope (APERIO CS2, Leica). Injury to the articular cartilage in the femur and tibia was assessed using the Modified Mankin score [35] on a 0-14 point scale, and using the Osteoarthritis Research Society International (OARSI) scale on a 0-24 point scale [36]. Because both tibial and femoral cartilage of left knee joints was evaluated, the maximum Mankin score was 28 and the maximum OARSI score was 48. In addition, we used microscopic scoring of synovium [37] to evaluate the histological changes of synovium. Two experienced observers (Yue Yang and Peng Shen) blindly scored samples.

\subsection{Gross imaging and computed tomography}

Knee joint images were captured by stereomicroscope (XTL-165-CB; Phenix), SkyScan 1276-CT (Bruker, Kontich, Belgium), and NRecon version 1.6 software (Bruker). Joint space narrowing, joint surface calcification, and articular cartilage damage were assessed. The degree of osteoarthritis was determined based on macro scores of surface roughness and erosion, according to the gross imaging scoring system described in previous literature; both the tibial and femoral joints were evaluated, with a maximum score of $10[36,38]$. Based on the OsteoArthritis Computed Tomography-Score (OACT-Score), joint space narrowing, osteophytosis, and subchondral cysts were used to quantify the degree of osteoarthritis detected by computed tomography. The tibiofemoral joint score was evaluated based on a range from 0 to 7 [39].

\subsection{Immunohistochemistry}

After tissue section deparaffinization and rehydration, serial sections were stained for collagen II (COLII) and matrix metalloproteinase (MMP)-13 using a two-step method following manufacturer instructions. Sections were incubated overnight at $4^{\circ} \mathrm{C}$ with rabbit polyclonal anti-COLII antibody (ab34712, 1:100; Abcam) and rabbit polyclonal anti-MMP-13 antibody (ab219620, 1:50; Abcam). The slides were then washed three times in PBS, incubated for 20 min at $37^{\circ} \mathrm{C}$ with an anti-mouse/rabbit IgG detection system (PV-9000, Zhongshan Goldenbridge Biotechnology Co., China), and stained with diaminobenzidine (DAB). Nuclei were counterstained with hematoxylin for $5 \mathrm{~min}$. The optical density of stained slides was measured using image analysis software (Nikon H600L Microscope and Image Analysis System, Japan). COLII was quantified by relative intensity. MMP-13 was quantified by the percentage of positive cells.

\subsection{Quantitative real-time polymerase chain reaction (qPCR)}

The qPCR protocol was conducted according to a previous study [22]. Expression levels were calculated using the $2^{-}$ $\triangle \triangle \mathrm{CT}$ method [40], with $\beta$-actin as the reference gene. Primer pair sequences were specific for rat CD86 (F: 5'- 
TCCTCCAGCAGTGGgAAACA-3'; R: 5'-TTTGTAGgTTTCGGGTATCCTTGC-3'), Arg1 (F: 5'-TTGATGTTGATGGACTGGAC-3'; R: 5'-TCTCTGGCTTATGATTACCTTC-3'), and ß-actin (F: 5'-GGAGATTACTGCCCTGGCTCCTA-3'; R: 5'GACTCATCGTACTCCTGCTTGCTG-3').

\subsection{Statistical analysis}

Data were analyzed using SPSS statistical software version 16 (SSPS, Inc., Chicago, IL, USA) and expressed as means with 95\% confidence intervals (Cls). Shapiro-Wilk's and Levene's tests were used to evaluate data normality and homogeneity, respectively. For variables that exhibited a normal distribution, independent sample t-tests and one-way ANOVA were used for statistical significance. A P value $<0.05$ was accepted as statistically significant.

\section{Results}

3.1 $\mathrm{LXA}_{4}$ is downregulated in articular fluid and CD 86/Arg 1 is upregulated in synovial tissue of patients with increasing Kellgren-Lawrence grade.

To evaluate the potential role of $\mathrm{LXA}_{4}$ and synovial macrophage M1/M2 subtype polarization in OA, we first detected the articular fluid and synovium samples divided into different Kellgren-Lawrence grades from 40 patients (Table 1). Western blots were evaluated for expression differences in CD86 (M1 macrophage marker) and Arg1 (M2 macrophage marker) [41] in patients with different of Kellgren-Lawrence grades. ELISA was evaluated for articular fluid expression differences for $\mathrm{LXA}_{4}$ in patients with different of Kellgren-Lawrence grades. We observed that CD 86/Arg 1 was upregulated in the synovial tissue (Fig. 1B), whereas $\mathrm{LXA}_{4}$ was downregulated in the articular fluid (Fig. 1C) of patients with increasing Kellgren-Lawrence grade.

3.2 CTS $(10 \%, 0.5 \mathrm{~Hz}, 24 \mathrm{~h})$ and $1 \mathrm{nM} \mathrm{LXA}$ promote synovial macrophage M2 subtype polarization.

To identify the best condition for further study, we treated synovial macrophage with different durations of CTS (10\%, $0.5 \mathrm{~Hz}$ ) and concentrations of $\mathrm{LXA}_{4}$ (Fig. 2B). At 24 and $48 \mathrm{~h} \mathrm{CTS}$, the percentages of CD-14-positive synovial macrophages and COL II-positive chondrocytes in the cultured cells exceeded 95\% (Fig. 2A). We observed a significant decrease in CD86 and increase in Arg 1 levels, but no significant changes were observed between 24 and 48 h CTS (Fig. 2C). Similarly, we observed a significant decrease in CD86 and increase in Arg1 expression with different LXA concentrations, with significant changes between $0.5 \mathrm{nM}$ and $1 \mathrm{nM} \mathrm{LXA}_{4}$. However, no significant change was seen in CD86 and Arg1 levels at 1, 2, and $4 \mathrm{nM} \mathrm{LXA}_{4}$ (Fig. 2D). Thus, we selected $24 \mathrm{~h}$ CTS $(10 \%, 0.5 \mathrm{~Hz})$ and $1 \mathrm{nM}$ LXA for subsequent analyses.

As shown in Fig. 3A, MMP-13 and CD86 levels were significantly decreased, and IL-10, TGF- $\beta 1$, VEGF receptor 1, and Arg 1 levels significantly increased in CTS $(24 \mathrm{~h}, 10 \%, 0.5 \mathrm{~Hz})$ - or LXA $(1 \mathrm{nM})$-induced synovial macrophages when compared to the control group. Moreover, combined CTS and LXA $(1 \mathrm{nM})$ exhibited synergism on synovial macrophages (Fig. 3A). ROS production in synovial macrophages was assessed by fluorescence microscopy and flow cytometry (Fig. 3B). CTS and LXA treatment resulted in significantly reduced ROS levels compared to the control (relative ROS intensity: Control = 1.00, 95\% Cl 0.94-1.06; CTS $(24 \mathrm{~h})=0.88,95 \% \mathrm{Cl} 0.82-0.95 ; \mathrm{LXA}_{4}(1 \mathrm{nM})=0.64,95 \%$ $\mathrm{Cl} 0.61-0.68 ; \mathrm{CTS}+\mathrm{LXA}_{4}=0.45,95 \% \mathrm{Cl} 0.38-0.51$.). Additionally, synovial macrophages treated with CTS and LXA exhibited significantly reduced apoptosis levels compared to those of the control (Fig. 3C).

3.3 CTS $(10 \%, 0.5 \mathrm{~Hz}, 24 \mathrm{~h})$ and $1 \mathrm{nM} \mathrm{LXA} 4$ inhibit chondrocyte pyroptosis via synovial macrophage M2 subtype polarization. 
We next explored the effects of CTS and $\mathrm{LXA}_{4}$ on chondrocytes in co-culture with synovial macrophages. Pro-IL-1 $\beta$, pro-

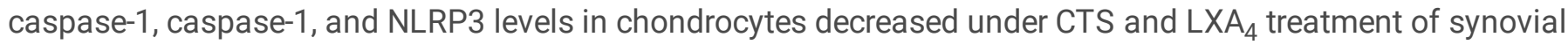
macrophage (Fig. 4B).

A considerable accumulation of NF-kB p65 occurred in chondrocyte nuclei in the control group. CTS and LXA suppressed this nuclear translocation under co-culture with synovial macrophages (Fig. 4C). As shown in Fig. 4D, TEM images show that chondrocytes from patients with OA exhibited a tendency for pyroptosis, including severe cytoplasmic edema, swelling of the cell membrane, decreased overall electron density of the cell matrix, and serious cavitation. However, LXA 4 CTS treatment of synovial macrophages inhibited the process of pyroptosis in the coculture.

\subsection{Treadmill exercise suppresses OA-related degeneration in MIA- induced rats}

40 male SD rats were divided into four groups (Fig. 5A). Gross imaging macroscopic score (Fig. 5B), histological assessments (Mankin and OARSI scores)(Fig. 5C and 5D), and computed tomography (OACT-Score) (Fig. 5E), histological changes of synovium (microscopic score) (Fig. 5F) indicated that cartilage and synovium damage in the OAG group was more severe than that in the CG group. OAE treatment appeared to exert therapeutic effects on tibiofemoral joint cartilage when compared with that of the OAG group. Therapeutic effects in the OAE group were suppressed by BOC-2 treatment (Fig. 5C, 5F). Immunohistochemical staining (COL II and MMP-13) revealed similar Mankin and OARSI scores (Fig. 5D).

TNF- $\alpha$ and IL-1 $\beta$ serum concentrations in the OAG were higher than those in the CG. The OAE group exhibited decreased TNF- $\alpha$ and IL-1 $\beta$ serum concentrations when compared with the OAG. However, the decrease in TNF- $\alpha$ and IL-1 $\beta$ concentrations was attenuated by BOC- 2 treatment. Changes in TNF- $\alpha$ and IL-1 $\beta$ concentrations in the IALF were similar to those in the serum levels. (Fig. 6B) Our data showed that the decrease of CD86 and the increase of Arg1 in synovial tissue were similar between the OAE group and the OAG. However, OAE effects were suppressed by BOC-2 (Fig. 6C). The relative expression levels of CD86 and Arg1 in synovial tissue as analyzed by qPCR were similar to those suggested by the western blot data (Fig. 6D).

\section{Discussion}

In this study, we evaluated the effects of $\mathrm{LXA}_{4}$ on chondrocyte pyroptosis via synovial macrophage $\mathrm{M} 2$ subtype polarization and identified the mechanisms by which it supports exercise therapy for OA.

Many studies have emphasized the importance of exercise therapy for $O A[25,26,42]$. However, most studies have focused on single cells, such as chondrocytes, synovial fibroblasts, or bone cells [29,30], and little is known about multi-cell co-culture in organisms. In our previous study, we demonstrated that $\mathrm{LXA}_{4}$ played an important role in exercise therapy for $\mathrm{OA}[11,12]$ and that moderate-intensity exercise had the best therapeutic effect [34, 43]. Furthermore, we previously demonstrated that exercise could act on cartilage through mechanical stress [33] and

altering the microenvironment around cartilage, such as cytokine LXA 4 in synovial fluid and synovial macrophages [44]. Therefore, it was important to understand mechanisms underlying mechanical stimulation and $\mathrm{LXA}_{4}$ function on synovial macrophages, and similarly, understand the molecular communications between synovial macrophages and chondrocytes. 
In our preliminary clinical experiment of the present study, we found that LXA 4 levels in articular fluid decreased and CD86/ Arg1 levels in synovial tissue increased with increasing Kellgren-Lawrence grade. In view of this finding, we predicted that $\mathrm{LXA}_{4}$ may affect the pathogenesis of osteoarthritis through macrophage polarization. Next, we verified our conjecture through in vitro experiments. According to previous experiments, we identified $\mathrm{LXA}_{4}$ as an exercise metabolite and an important factor in the exercise treatment of OA [11, 12], and we used CTS to simulate the microenvironment of exercise on chondrocytes and macrophages $[33,45]$. We found that CTS supported by LXA treatment inhibited chondrocyte pyroptosis via synovial macrophage M2 subtype polarization. In our in vivo experiments, we established treadmill exercise protocol to validate the data collected in vitro. Our data showed that CD86 levels decreased and Arg1 levels increased in a manner consistent with that seen in the human clinical data. Based on these results, we concluded that $\mathrm{LXA}_{4}$ administration can support CTS therapy via synovial macrophage M2 subtype polarization, offering a new potential treatment for osteoarthritis.

Intra-articular injection of MIA in rats, which caused histological changes, such as cartilage surface erosion, matrix loss, and synovitis, is considered an accurate model of OA [38]. Our findings showed that therapeutic effects of moderateintensity treadmill exercise were suppressed by BOC-2, an antagonist of the LXA $\mathrm{A}_{4}$ receptor which confirmed the results of our previous study [11]. The M2 polarization of macrophages is considered significant in treating various diseases. Although the positive effects of M2 polarization on synovial macrophages in OA have been reported [46], molecular mechanistic outputs from exercise therapy on synovial macrophages are unclear. We studied CD86, a marker of M1 macrophage, and Arg1, a marker of M2 macrophage [41] expression in the synovium of different experimental groups. Both western blotting and qPCR data showed decreased CD86 and increased Arg1 expression in synovium in the OAE group when compared with that of the OAG. These effects were suppressed by BOC-2 treatment.

The mechanisms underlying the conversion of biomechanical signals into intercellular events have become a major focus in OA research. Although synovial macrophages undergo mechanical stimulation during exercise therapy, little is known about how this stimulation regulates the immune response. Accordingly, mechanical stress may play a role in macrophage recruitment or polarization [47]. We showed that CTS initiated an inflammatory response similar to that induced in the joints during exercise, via the M2 polarization of synovial macrophages. Our data (Fig. 5C and 5D) indicated that CTS $(10 \%, 0.5 \mathrm{~Hz})$ for $24 \mathrm{~h}$ and $\mathrm{LXA}_{4}$ administration $(1 \mathrm{nM})$ decreased CD86 expression and increased Arg1 expression, causing M2 polarization of synovial macrophages. However, CTS $(10 \%, 0.5 \mathrm{~Hz})$ over $48 \mathrm{~h}$ exerted the opposite effects. Thus, we selected CTS $(10 \%, 0.5 \mathrm{~Hz}, 24 \mathrm{~h})$ and $\mathrm{LXA}_{4}(1 \mathrm{nM})$ for subsequent experiments. Our results showed these parameters significantly decreased ROS, MMP-13, and CD86 expression in synovial macrophages. Taken together, these findings demonstrated that CTS $(10 \%, 0.5 \mathrm{~Hz}, 24 \mathrm{~h})$ and $\mathrm{LXA}_{4}(1 \mathrm{nM})$ stimulated multiple chemokines, such as IL-10, TGF- $\beta 1$, VEGF receptor 1 and Arg1, to facilitate macrophage M2 polarization. Equally, LXA4 and CTS stimulation also reduced apoptosis in synovial macrophages via M2 polarization. There is much evidence supporting physical and mechanical regulation of macrophage phenotype polarization and function.

Biophysical cues can function synergistically with soluble factors to mediate chondrocyte behavior [48]. The links between force distribution, tissue damage, morphogen gradients, and cell responses have garnered substantial research interest. We discovered the co-culture of synovial macrophages and chondrocytes by transwell chamber. Mechanical stimulation can induce alternative activation of macrophages, which have functional roles in chondrocyte regeneration. We further revealed that IL-10, TGF $\beta 1$, VEGF receptor 1, and Arg 1, which are produced by M2 subtype macrophages, were functional mediators in OA exercise therapy. Our work suggested that CTS and $\mathrm{LXA}_{4}$ are parameters of synovial macrophage M1/M2 polarization, and revealed an unexpected complexity in the co-culture of synovial macrophages and chondrocytes. Moderate CTS and LXA $\mathrm{LA}_{4}$ treatment showed a synergistic effect on promoting M2 polarization in synovial macrophages, which inhibited the nuclear translocation of NF-kB p65, forming NLRP3 in chondrocytes. 
Our previous studies showed that $L X A_{4}$ was rapidly produced and metabolized [11, 12], suggesting that LXA 4 plays an important role in exercise therapy for OA. Because there is no $L_{X A}$ receptor (ALX/FPR2) in chondrocytes, $L_{4} A_{4}$ cannot directly act on these cells. In the knee joint, $L_{X A_{4}}$ combines with synovial macrophages, fibroblasts, and neutrophils to reduce chronic inflammation, generating therapeutic effects for OA $[49,50]$. The effects of exercise on $0 A$ may be divided into systemic effects on the whole body and local effects on the knee joint. Appropriate exercise produces a hypoxic environment in blood vessels which promote platelet aggregation, lipoxygenase activation, and LXA generation [9]. It is proposed that $\mathrm{LXA}_{4}$ not only prevents chronic inflammation and limits neutrophil infiltration, but also relieves pain, reduces pain sensitivity, and inhibits peripheral nerve fiber sensitivity in systemic system of whole body $[51,52]$. Additionally, appropriate exercise may generate synovial fluid and lipids in the infrapatellar fat pad, producing $\mathrm{LXA}_{4}$ in the knee joint. LXA 4 may act on synovial macrophages to promote M2 polarization, and act on synovial fibroblasts to reduce MMPs and a disintegrin and metalloproteinase with thrombospondin motifs [11], thus relieving synovitis. Thus, our study identified a strategy whereby chondrocyte regeneration via synovial macrophage M2 subtype polarization can be encouraged via exercise therapy and $\mathrm{LXA}_{4}$ treatment (Fig. 7).

Our study has several limitations that should be noted. First, due to ethical limitations, we were able to sample cartilage from OA patients with different Kellgren-Lawrence grades. Secondly, the MIA-induced OA rat model may not have fully represented all aspects of OA. This model involved measurements as a single time point, presenting a chance of random effects. We plan to study other OA models and analyze them in combination with the MIA model. Thirdly, we used BOC-2 as an antagonist of the LXA receptor. It is well known that chemical inhibitors can produce off-target effects. Our research group has begun to conduct gene knockdown experiments to further study this problem. Fourth, in our previous studies, we demonstrated that moderate-intensity treadmill exercise alleviated MIA-induced OA in rats [11, 12], and that moderate CTS $(10 \%, 0.5 \mathrm{~Hz}, 4 \mathrm{hr})$ ameliorated the degeneration of cartilage and chondrocytes [33, 45]. Because the main effect of exercise on articular cartilage is mechanical stimulation [53], we aim to study the similarities between CTS and treadmill exercise in animals in subsequent experiments, but the moderate CTS cannot be directly transformed into activities or common exercises OA patients may participate in. Finally, western blotting of whole synovial tissue is a very crude measure of CD86/Arg1, which could be expressed by numerous cell types in addition to macrophages and be influenced by other cells present in the synovial tissue. In this paper, we focused on the influence of synovial macrophages, and aim to study the role of other cells in this process in future experiments.

In summary, our work characterized mechanical stimulation and M2 polarization of the synovial macrophagechondrocyte regeneration axis in response to $\mathrm{LXA}_{4}$, and demonstrated how organs can regenerate in response to exercise therapy. We used treadmill exercises of MIA-induced OA rats, CTS and LXA 4 responses on synovial macrophages, and the co-culture of synovial macrophages and chondrocytes to explore their effects on OA. Our findings indicated that CTS and $\mathrm{LXA}_{4}$ inhibited the nuclear translocation of NF-kB p65 and formation of NLRP3 in chondrocytes via $\mathrm{M} 2$ polarization of synovial macrophages. Our results identified the effects of mechanical stress and $\mathrm{LXA}_{4}$ on synovial macrophages, and provided evidence of molecular signaling between synovial macrophages and chondrocytes during exercise therapy.

\section{Declarations}

\section{Statements and Declarations}

\section{FUNDING}


This study was supported by the National Natural Science Foundation of China (Grant No. 82102613), and the Youth Science and Technology Talent Project of Liaoning Provincial Education Department (Grant No. QNZR2020014), and the 345 Talent Project of Shengjing Hospital of China Medical University.

\section{CONFLICTS OF INTEREST}

The authors declare that they have no competing interests.

\section{AUTHORS' CONTRIBUTIONS}

Yue Yang and Peng Shen and Shuangshuo Jia contributed to study conception and design. Yue Yang, Yang Wang, Xiaonan Zhou, and Delong Zhang conducted treadmill exercise experiments. Yue Yang, Shuangshuo Jia, Yang Wang, Zhuangzhuang Jin, Ziyuan Wang, and Donghao Liu conducted cell culture and cyclic tensile strain experiments. All authors contributed to data acquisition and analysis. Yue Yang, Yang Wang, Lunhao Bai, Peng Shen and Shuangshuo Jia conducted statistical analysis and prepared the manuscript. Yue Yang, Peng Shen and Shuangshuo Jia approved of the final draft. Peng Shen and Shuangshuo Jia are co-first authors of the article. All authors contributed to critical manuscript revision for intellectual content and approved the manuscript for publication.

\section{data availability statement}

All data generated or analysed during this study are included in this published article.

\section{ETHICS STATEMENT}

This study was carried out under the approval and in accordance with the guidelines of the Ethics Committee of Shengjing Hospital of China Medical University.

\section{Informed Consent}

Informed consent was obtained from all individual participants included in the study.

\section{References}


1. Hunter DJ, Bierma-Zeinstra S (2019) Osteoarthr Lancet (London England) 393(10182):1745-1759. https://doi.org/10.1016/s0140-6736(19)30417-9

2. Ondrésik M, Azevedo Maia FR, da Silva Morais A, Gertrudes AC, Dias Bacelar AH, Correia C, Gonçalves C, Radhouani H, Amandi Sousa R, Oliveira JM, Reis RL (2017) Management of knee osteoarthritis. Current status and future trends. Biotechnol Bioeng 114(4):717-739. https://doi.org/10.1002/bit.26182

3. Wallace IJ, Worthington S, Felson DT, Jurmain RD, Wren KT, Maijanen H, Woods RJ, Lieberman DE (2017) Knee osteoarthritis has doubled in prevalence since the mid-20th century. Proc Natl Acad Sci USA 114(35):9332-9336. https://doi.org/10.1073/pnas.1703856114

4. Hulshof CTJ, Colosio C, Daams JG, Ivanov ID, Prakash KC, Kuijer P, Leppink N, Mandic-Rajcevic S, Masci F, van der Molen HF, Neupane S, Nygård CH, Oakman J, Pega F, Proper K, Prüss-Üstün AM, Ujita Y, Frings-Dresen MHW (2019) WHO/ILO work-related burden of disease and injury: Protocol for systematic reviews of exposure to occupational ergonomic risk factors and of the effect of exposure to occupational ergonomic risk factors on osteoarthritis of hip or knee and selected other musculoskeletal diseases. Environ Int 125:554-566.

https://doi.org/10.1016/j.envint.2018.09.053

5. Serhan CN, Levy BD (2018) Resolvins in inflammation: emergence of the pro-resolving superfamily of mediators. J Clin Investig 128(7):2657-2669. https://doi.org/10.1172/jci97943

6. Delanois RE, Etcheson JI, Sodhi N, Henn RF 3, Gwam CU, George NE, Mont MA (2019) Biologic Therapies for the Treatment of Knee Osteoarthritis. J Arthroplast 34(4):801-813. https://doi.org/10.1016/j.arth.2018.12.001

7. Serhan CN, Chiang N, Dalli J (2018) New pro-resolving n-3 mediators bridge resolution of infectious inflammation to tissue regeneration, Molecular aspects of medicine 64. 1-17. https://doi.org/10.1016/j.mam.2017.08.002

8. Conaghan PG, Cook AD, Hamilton JA, Tak PP (2019) Therapeutic options for targeting inflammatory osteoarthritis pain. Nat Rev Rheumatol 15(6):355-363. https://doi.org/10.1038/s41584-019-0221-y

9. McMahon B, Mitchell S, Brady HR, Godson C (2001) Lipoxins: revelations on resolution. Trends Pharmacol Sci 22(8):391-395. https://doi.org/10.1016/s0165-6147(00)01771-5

10. Ke Y, Zebda N, Oskolkova O, Afonyushkin T, Berdyshev E, Tian Y, Meng F, Sarich N, Bochkov VN, Wang JM, Birukova AA, Birukov KG (2017) Anti-Inflammatory Effects of OxPAPC Involve Endothelial Cell-Mediated Generation of LXA4. Circul Res 121(3):244-257. https://doi.org/10.1161/circresaha.116.310308

11. Yang Y, Wang Y, Kong Y, Zhang X, Zhang H, Gang Y, Bai L (2018) The therapeutic effects of lipoxin A4 during treadmill exercise on monosodium iodoacetate-induced osteoarthritis in rats. Mol Immunol 103:35-45. https://doi.org/10.1016/j.molimm.2018.08.027

12. Yang $Y$, Wang $Y$, Kong Y, Zhang X, Bai L (2017) The effects of different frequency treadmill exercise on lipoxin A4 and articular cartilage degeneration in an experimental model of monosodium iodoacetate-induced osteoarthritis in rats. PLoS ONE 12(6):e0179162. https://doi.org/10.1371/journal.pone.0179162

13. Millerand M, Berenbaum F, Jacques $C$ (2019) Danger signals and inflammaging in osteoarthritis, Clinical and experimental rheumatology 37 Suppl 120(5)48-56

14. Wood MJ, Leckenby A, Reynolds G, Spiering R, Pratt AG, Rankin KS, Isaacs JD, Haniffa MA, Milling S, Hilkens CM (2019) Macrophage proliferation distinguishes 2 subgroups of knee osteoarthritis patients. JCl insight 4(2). https://doi.org/10.1172/jci.insight.125325

15. Smith MD, Barg E, Weedon H, Papengelis V, Smeets T, Tak PP, Kraan M, Coleman M, Ahern MJ (2003) Microarchitecture and protective mechanisms in synovial tissue from clinically and arthroscopically normal knee joints. Ann Rheum Dis 62(4):303-307. https://doi.org/10.1136/ard.62.4.303

16. Daghestani HN, Pieper CF, Kraus VB, Hoboken (2015) N J) 67(4):956-965. https://doi.org/10.1002/art.39006 
17. Choi MC, Jo J, Park J, Kang HK, Park Y (2019) NF-кB Signaling Pathways in Osteoarthritic Cartilage Destruction. Cells 8(7). https://doi.org/10.3390/cells8070734

18. Culemann S, Grüneboom A, Nicolás-Ávila J, Weidner D, Lämmle KF, Rothe T, Quintana JA, Kirchner P, Krljanac B, Eberhardt M, Ferrazzi F, Kretzschmar E, Schicht M, Fischer K, Gelse K, Faas M, Pfeifle R, Ackermann JA, Pachowsky M, Renner N, Simon D, Haseloff RF, Ekici AB, Bäuerle T, Blasig IE, Vera J, Voehringer D, Kleyer A, Paulsen F, Schett G, Hidalgo A, Krönke G (2019) Locally renewing resident synovial macrophages provide a protective barrier for the joint. Nature 572(7771):670-675. https://doi.org/10.1038/s41586-019-1471-1

19. Sica A, Mantovani A (2012) Macrophage plasticity and polarization: in vivo veritas. J Clin Investig 122(3):787-795. https://doi.org/10.1172/jci59643

20. Mills CD, Macrophages M (2012) Oracles of Health and Disease. Crit Rev Immunol 32(6):463-488. https://doi.org/10.1615/critrevimmunol.v32.i6.10

21. Mills CD, Ley K (2014) M2 macrophages: the chicken and the egg of immunity. J Innate Immun 6(6):716-726. https://doi.org/10.1159/000364945

22. Mills CD (2015) Anatomy of a discovery: m1 and m2 macrophages. Front Immunol 6:212. https://doi.org/10.3389/fimmu.2015.00212

23. Kesavardhana S, Malireddi RKS, Kanneganti TD (2020) Caspases in Cell Death, Inflammation, and Pyroptosis. Annu Rev Immunol 38:567-595. https://doi.org/10.1146/annurev-immunol-073119-095439

24. Hu Y, Li Y, Hu H, An S (2020) Pyroptosis Plays a Role in Osteoarthritis. Aging and disease 11(5):1146. https://doi.org/10.14336/ad.2019.1127

25. Abbasi J (2017) Can Exercise Prevent Knee Osteoarthritis? JAMA 318(22):2169-2171. https://doi.org/10.1001/jama.2017.16144

26. Emery CA, Pasanen K (2019) Current trends in sport injury prevention, Best practice \& research. Clin Rheumatol 33(1):3-15. https://doi.org/10.1016/j.berh.2019.02.009

27. Wellsandt E, Golightly Y (2018) Exercise in the management of knee and hip osteoarthritis. Curr Opin Rheumatol 30(2):151-159. https://doi.org/10.1097/bor.0000000000000478

28. Chu SY, Chou CH, Huang HD, Yen MH, Hong HC, Chao PH, Wang YH, Chen PY, Nian SX, Chen YR, Liou LY, Liu YC, Chen HM, Lin FM, Chang YT, Chen CC, Lee OK (2019) Mechanical stretch induces hair regeneration through the alternative activation of macrophages. Nat Commun 10(1):1524. https://doi.org/10.1038/s41467-019-09402-8

29. Yang Y, Wang Y, Kong Y, Zhang X, Zhang H, Gang Y, Bai L (2019) Mechanical stress protects against osteoarthritis via regulation of the AMPK/NF-KB signaling pathway. J Cell Physiol 234(6):9156-9167.

https://doi.org/10.1002/jcp.27592

30. Yu HS, Kim JJ, Kim HW, Lewis MP, Wall I (2016) Impact of mechanical stretch on the cell behaviors of bone and surrounding tissues. J tissue Eng 7:2041731415618342. https://doi.org/10.1177/2041731415618342

31. Wu CL, Harasymowicz NS, Klimak MA, Collins KH, Guilak F (2020) The role of macrophages in osteoarthritis and cartilage repair. Osteoarthr Cartil 28(5):544-554. https://doi.org/10.1016/j.joca.2019.12.007

32. Iliopoulos D, Malizos KN, Tsezou A (2007) Epigenetic regulation of leptin affects MMP-13 expression in osteoarthritic chondrocytes: possible molecular target for osteoarthritis therapeutic intervention. Ann Rheum Dis 66(12):1616-1621. https://doi.org/10.1136/ard.2007.069377

33. Yang Y, Wang Y, Kong Y, Zhang X, Zhang H, Gang Y, Bai L (2019) Mechanical stress protects against osteoarthritis via regulation of the AMPK/NF-kappaB signaling pathway. J Cell Physiol 234(6):9156-9167.

https://doi.org/10.1002/jcp.27592

Page $14 / 23$ 
34. Li Z, Huang Z, Zhang H, Lu J, Tian Y, Piao S, Lin Z, Bai L (2021) Moderate-intensity exercise alleviates pyroptosis by promoting autophagy in osteoarthritis via the P2X7/AMPK/mTOR axis. Cell Death Discov 7(1):346.

https://doi.org/10.1038/s41420-021-00746-z

35. Pritzker KP, Gay S, Jimenez SA, Ostergaard K, Pelletier JP, Revell PA, Salter D, van den Berg WB (2006) Osteoarthritis cartilage histopathology: grading and staging. Osteoarthr Cartil 14(1):13-29.

https://doi.org/10.1016/j.joca.2005.07.014

36. Gerwin N, Bendele AM, Glasson S, Carlson CS (2010) The OARSI histopathology initiative - recommendations for histological assessments of osteoarthritis in the rat, Osteoarthritis and cartilage 18 Suppl 3. S24-34. https://doi.org/10.1016/j.joca.2010.05.030

37. Glasson SS, Chambers MG, Van Den Berg WB, Little CB (2010) The OARSI histopathology initiative recommendations for histological assessments of osteoarthritis in the mouse. Osteoarthr Cartil 18 Suppl 3S1723. https://doi.org/10.1016/j.joca.2010.05.025

38. Lin Z, Miao J, Zhang T, He M, Wang Z, Feng X, Bai L (2021) JUNB-FBXO21-ERK axis promotes cartilage degeneration in osteoarthritis by inhibiting autophagy. Aging Cell 20(2). https://doi.org/10.1111/acel.13306

39. Gielis WP, Weinans H, Nap FJ, Roemer FW, Foppen W (2020) Scoring Osteoarthritis Reliably in Large Joints and the Spine Using Whole-Body CT: OsteoArthritis Computed Tomography-Score (OACT-Score). J Pers Med 11(1). https://doi.org/10.3390/jpm11010005

40. Livak KJ, Schmittgen TD (2001) Analysis of relative gene expression data using real-time quantitative PCR and the 2(-Delta Delta C(T)) Method, Methods (San Diego. Calif) 25(4):402-408. https://doi.org/10.1006/meth.2001.1262

41. Lee H, Kim H, Seo J, Choi K, Lee Y, Park K, Kim S, Mobasheri A, Choi H (2020) TissueGene-C promotes an antiinflammatory micro-environment in a rat monoiodoacetate model of osteoarthritis via polarization of M2 macrophages leading to pain relief and structural improvement. Inflammopharmacology 28(5):1237-1252. https://doi.org/10.1007/s10787-020-00738-y

42. Kolasinski SL, Neogi T, Hochberg MC, Oatis C, Guyatt G, Block J, Callahan L, Copenhaver C, Dodge C, Felson D, Gellar K, Harvey WF, Hawker G, Herzig E, Kwoh CK, Nelson AE, Samuels J, Scanzello C, White D, Wise B, Altman RD, DiRenzo D, Fontanarosa J, Giradi G, Ishimori M, Misra D, Shah AA, Shmagel AK, Thoma LM, Turgunbaev M, Turner AS, Reston J (2020) 2019 American College of Rheumatology/Arthritis Foundation Guideline for the Management of Osteoarthritis of the Hand, Hip, and Knee. Arthritis Care Res 72(2):149-162. https://doi.org/10.1002/acr.24131

43. Zhang H, Ji L, Yang Y, Wei Y, Zhang X, Gang Y, Lu J, Bai L (2019) IF3.2The Therapeutic Effects of Treadmill Exercise on Osteoarthritis in Rats by Inhibiting the HDAC3/NF-KappaB Pathway in vivo and in vitro. Front Physiol 10:1060. https://doi.org/10.3389/fphys.2019.01060

44. Akhbari P, Karamchandani U, Jaggard MKJ, Graça G, Bhattacharya R, Lindon JC, Williams HRT, Gupte CM (2020) Can joint fluid metabolic profiling (or "metabonomics") reveal biomarkers for osteoarthritis and inflammatory joint disease?: A systematic review. Bone \& joint research 9(3):108-119. https://doi.org/10.1302/2046-3758.93.bjr2019-0167.r1

45. Yang Y, Wang Y, Kong Y, Zhang X, Zhang H, Feng X, Wang Z, Gao P, Yan M, Bai L, Li F (2020) Moderate Mechanical Stimulation Protects Rats against Osteoarthritis through the Regulation of TRAIL via the NF-kappaB/NLRP3 Pathway. Oxid Med Cell Longev 2020:6196398. https://doi.org/10.1155/2020/6196398

46. Hu Y, Gui Z, Zhou Y, Xia L, Lin K, Xu Y (2019) Quercetin alleviates rat osteoarthritis by inhibiting inflammation and apoptosis of chondrocytes, modulating synovial macrophages polarization to M2 macrophages, Free radical biology \& medicine 145. 146-160. https://doi.org/10.1016/j.freeradbiomed.2019.09.024

47. Adams S, Wuescher LM, Worth R, Yildirim-Ayan E (2019) Mechano-Immunomodulation: Mechanoresponsive Changes in Macrophage Activity and Polarization. Ann Biomed Eng 47(11):2213-2231.

Page $15 / 23$ 
https://doi.org/10.1007/s10439-019-02302-4

48. Vaca-González JJ, Guevara JM, Moncayo MA, Castro-Abril H, Hata Y (2019) Garzón-Alvarado, Biophysical Stimuli: A Review of Electrical and Mechanical Stimulation in Hyaline Cartilage. Cartilage 10(2):157-172. https://doi.org/10.1177/1947603517730637

49. Serhan CN (2014) Pro-resolving lipid mediators are leads for resolution physiology. Nature 510(7503):92-101. https://doi.org/10.1038/nature13479

50. Maderna P, Godson C (2009) Lipoxins: resolutionary road. Br J Pharmacol 158(4):947-959. https://doi.org/10.1111/j.1476-5381.2009.00386.x

51. Wang C, Xiao M, Liu X, Ni C, Liu J, Erben U, Qin Z (2013) IFN-y-mediated downregulation of LXA4 is necessary for the maintenance of nonresolving inflammation and papilloma persistence. Cancer Res 73(6):1742-1751. https://doi.org/10.1158/0008-5472.can-12-2801

52. Mei HX, Ye Y, Xu HR, Xiang SY, Yang Q, Ma HY, Jin SW, Wang Q (2021) LXA4 Inhibits Lipopolysaccharide-Induced Inflammatory Cell Accumulation by Resident Macrophages in Mice. J Inflamm Res 14:1375-1385. https://doi.org/10.2147/jir.s301292

53. Buckwalter JA, Anderson DD, Brown TD, Tochigi Y, Martin JA (2013) The Roles of Mechanical Stresses in the Pathogenesis of Osteoarthritis: Implications for Treatment of Joint Injuries. Cartilage 4(4):286-294. https://doi.org/10.1177/1947603513495889

\section{Table 1}

Table 1. Baseline characteristics of patients

\begin{tabular}{|c|c|c|c|c|c|c|c|c|}
\hline $\begin{array}{l}\text { Basic } \\
\text { information }\end{array}$ & & $\begin{array}{l}\text { Kellgren- } \\
\text { Lawrence } \\
\text { grade } 0\end{array}$ & $\begin{array}{l}\text { Kellgren- } \\
\text { Lawrence } \\
\text { grade } 1\end{array}$ & $\begin{array}{l}\text { Kellgren- } \\
\text { Lawrence } \\
\text { grade } 2\end{array}$ & $\begin{array}{l}\text { Kellgren- } \\
\text { Lawrence } \\
\text { grade } 3\end{array}$ & $\begin{array}{l}\text { Kellgren- } \\
\text { Lawrence } \\
\text { grade } 4\end{array}$ & Statistic & $\begin{array}{l}P \\
\text { value }\end{array}$ \\
\hline \multirow{2}{*}{$\begin{array}{l}\text { Biological } \\
\text { Sex }\end{array}$} & Male & 13 & 15 & 12 & 15 & 12 & $c^{2}=5.501$ & 0.240 \\
\hline & Female & 15 & 18 & 19 & 28 & 37 & & \\
\hline \multirow[t]{2}{*}{ Age (years) } & $\nabla 65$ & 28 & 33 & 16 & 8 & 5 & $c^{2}=108.99$ & $<0.001$ \\
\hline & $\geq 65$ & 0 & 0 & 15 & 35 & 44 & & \\
\hline \multirow[t]{4}{*}{$\begin{array}{l}\text { Obesity } \\
\text { gradation }\end{array}$} & $\begin{array}{l}\text { Under } \\
\text { weight }^{\mathrm{a}}\end{array}$ & 2 & 1 & 0 & 1 & 2 & $c^{2}=52.113$ & $<0.001$ \\
\hline & $\begin{array}{l}\text { Normal } \\
\text { weight }\end{array}$ & 22 & 27 & 21 & 24 & 10 & & \\
\hline & Overweight & 4 & 4 & 8 & 15 & 23 & & \\
\hline & Obesity & 0 & 1 & 2 & 3 & 14 & & \\
\hline
\end{tabular}

aUnderweight: $\mathrm{BMI}<18.5$; Normal weight: $18.5 \leq \mathrm{BMI}<24.9$; Overweight: $25 \leq \mathrm{BMI}<30$; Obesity: $\mathrm{BMI} \geq 30$.

\section{Figures}


A

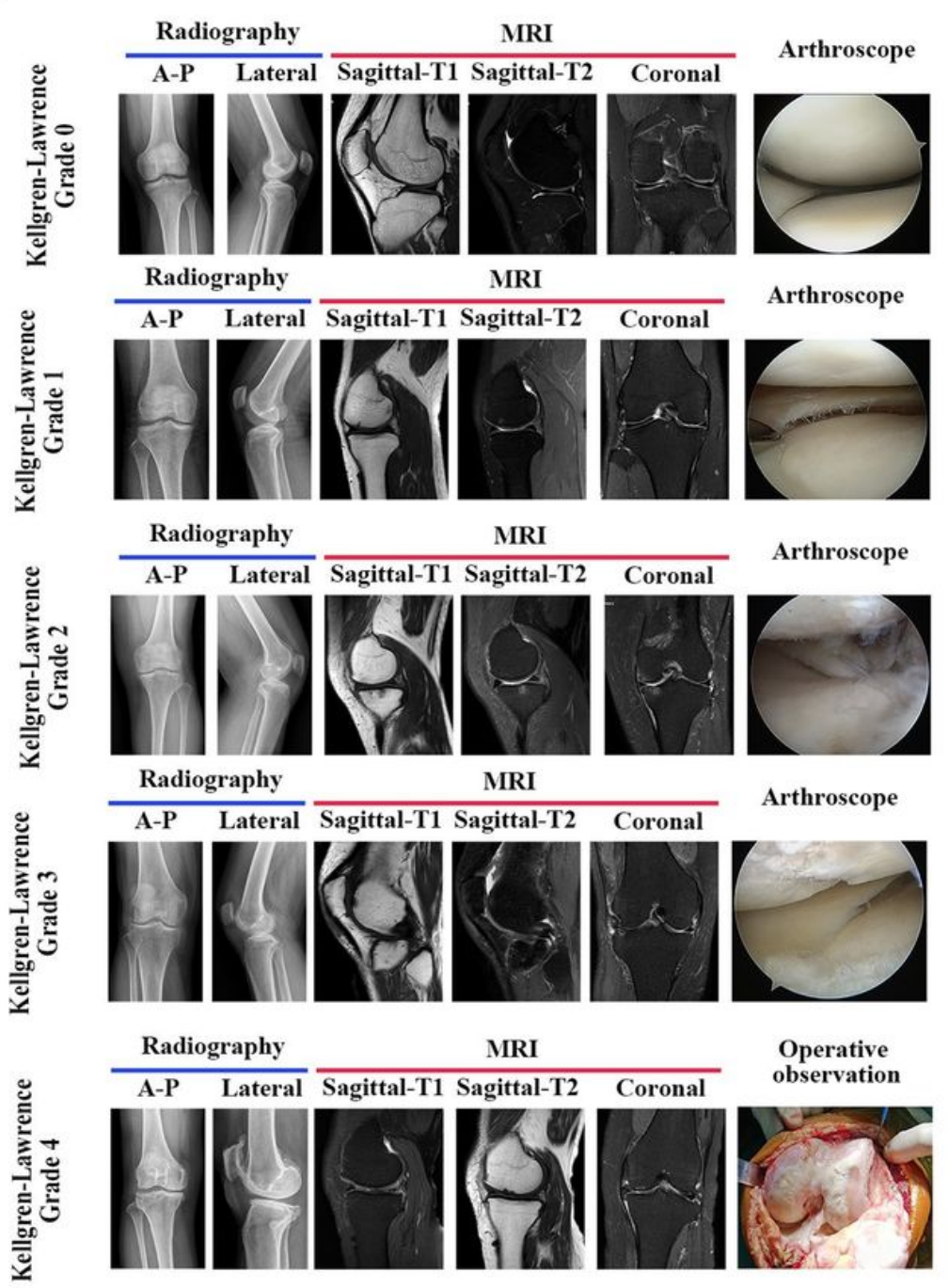

B
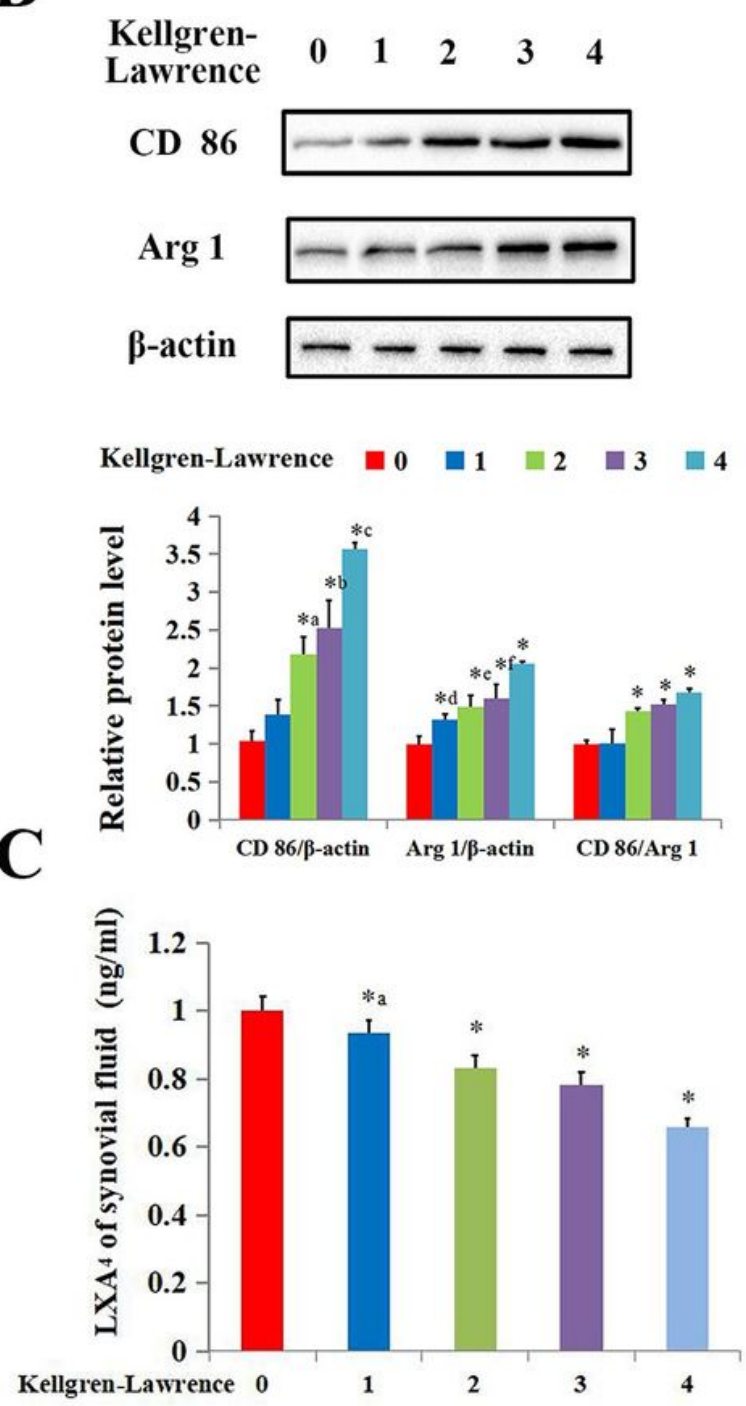

Figure 1

Expression of $\mathrm{LXA}_{4}$ is downregulated in articular fluid and CD 86/Arg 1 is upregulated in synovial tissue with increasing Kellgren-Lawrence grade (A) Plain radiograph and magnetic resonance imaging (MRI) scans. A-P: anterior-posterior. (B) Western blots of CD 86 and Arg 1 in synovial tissue. Comparison of all Kellgren-Lawrence grades against grade 0 $\left({ }^{\star} P<0.001,{ }^{{ }^{*} \mathrm{a}} P=0.002,{ }^{\text {bb }} P=0.002,{ }^{{ }^{\circ}} P=0.003,{ }^{\star} \mathrm{d} P=0.003,{ }^{*} \mathrm{e} P=0.008,{ }^{* \mathrm{f}} P=0.015\right.$; ANOVA) shown. $\beta$-actin was used as an internal control. Data are presented as means $\pm 95 \% \mathrm{Cl} ; n=$ three patients per group. (C) ELISA results of LXA in articular fluid of patients. Differences between CG and OAG $\left({ }^{\star} P<0.001,{ }^{\star}{ }^{a} P=0.008\right.$; ANOVA) shown. Data are presented as means $\pm 95 \% \mathrm{Cl} ; \mathrm{n}=$ eight patients per group. 
A
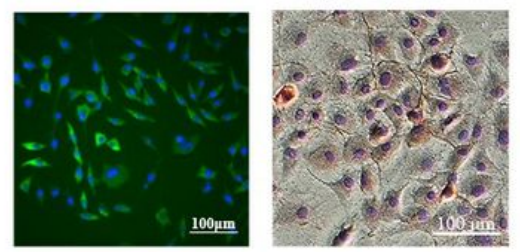

C

CTS(h) $\quad 0 \quad 6 \quad 12 \quad 2448 \quad 7296$

CD 86 -

Arg 1

$\beta$-actin

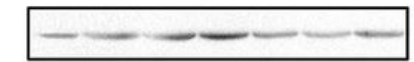

D
B

synovial macrophage with different concentration of LXA

culture of synovial macrophage (OA patient)
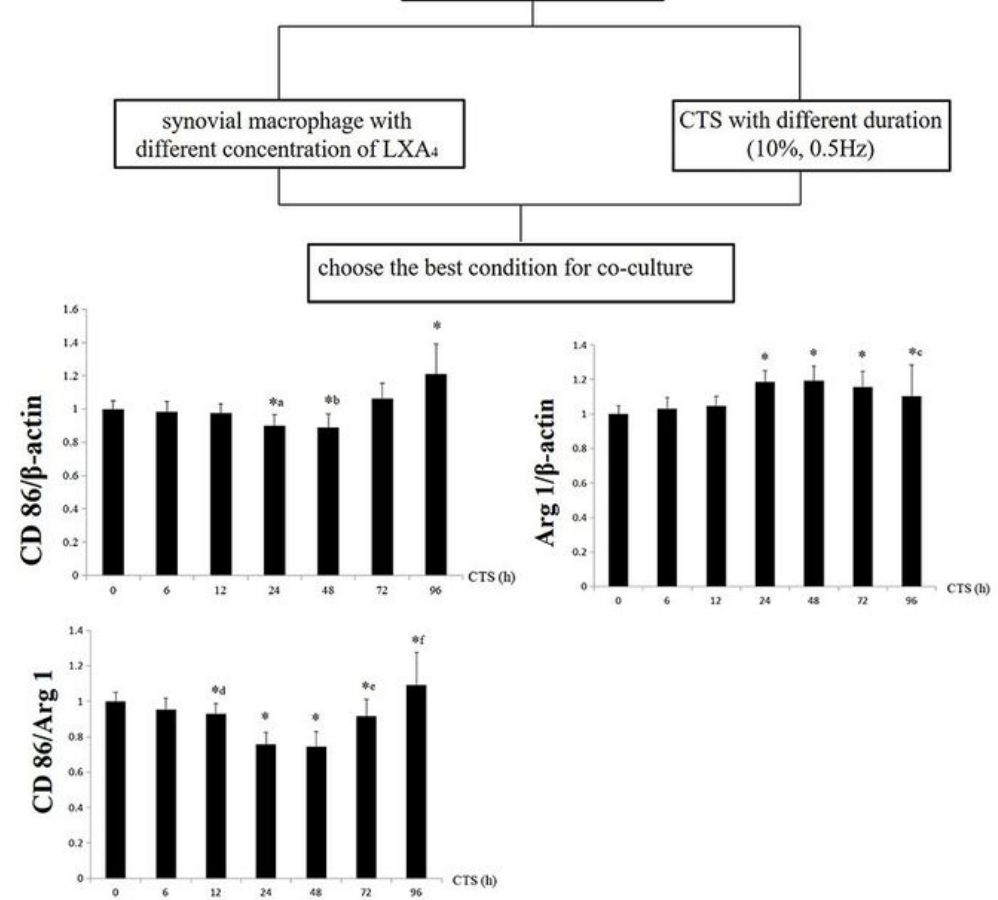

$\begin{array}{llllll}\mathrm{LXA}_{4}(\mathrm{nM}) & 0 & 0.5 & 1 & 2 & 4\end{array}$

CD 86

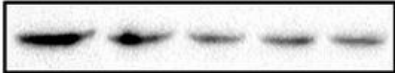

$\operatorname{Arg} 1$

$\beta$-actin

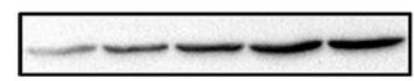

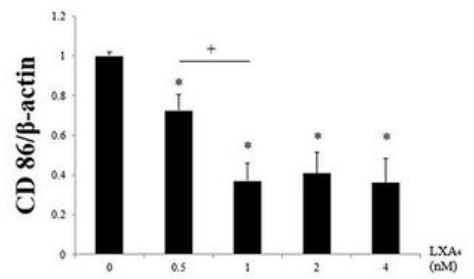
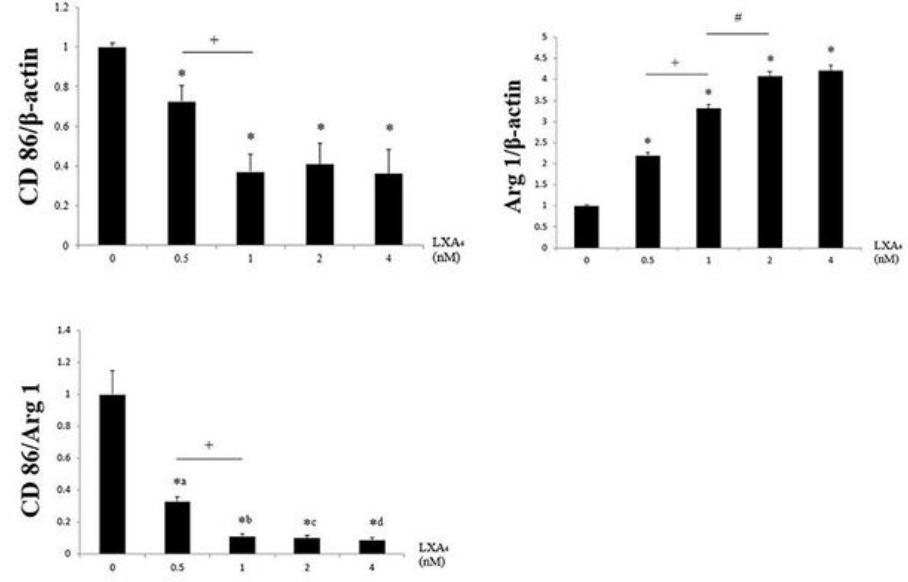

Figure 2

Western blotting of synovial macrophages treated with CTS and $\mathrm{LXA}_{4}(\mathrm{~A})$ Representative immunohistochemical image of chondrocytes stained for COL II (brown) and synovial macrophages stained for CD14 (green). The percentage of CD14-positive synovial macrophages and that of COL II-positive chondrocytes in the cultured cells was more than $95 \%$. Scale bar $=100 \mu \mathrm{m}$. (B) Synovial macrophage experimental design with CTS and LXA . (C) Synovial macrophages were subjected to CTS $(10 \%, 0.5 \mathrm{~Hz})$ for different durations $(0 \mathrm{~h}, 6 \mathrm{~h}, 12 \mathrm{~h}, 24 \mathrm{~h}, 48 \mathrm{~h}, 72 \mathrm{~h}, 96 \mathrm{~h})$. Differences between

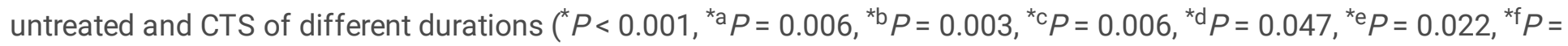
0.011) were significant (ANOVA). Data are presented as means $\pm 95 \% \mathrm{Cl} ; n=$ three per group. (D) Synovial macrophages were subjected to $\mathrm{LXA}_{4}$ at different concentrations $(0,0.5 \mathrm{nM}, 1 \mathrm{nM}, 2 \mathrm{nM}, 4 \mathrm{nM})$. Differences between untreated and $\mathrm{LXA}_{4}$ for different concentration $\left({ }^{\mathrm{k}} P<0.001,{ }^{{ }^{*} \mathrm{a}} P=0.020,{ }^{{ }_{\mathrm{b}}} P=0.013,{ }^{{ }^{*} \mathrm{c}} P=0.012,{ }^{{ }_{\mathrm{d}}} P=0.012\right)$, differences between 
$\operatorname{LXA}_{4}(0.5 \mathrm{nM})$ and $\mathrm{LXA}_{4}(1 \mathrm{nM})\left({ }^{+} P<0.001\right)$, and differences between $\mathrm{LXA}_{4}(1 \mathrm{nM})$ and $\mathrm{LXA}_{4}(2 \mathrm{nM})\left({ }^{+} P<0.001\right)$ were significant (ANOVA). Data are presented as means $\pm 95 \% \mathrm{Cl} ; \mathrm{n}=$ three per group.

\section{A}
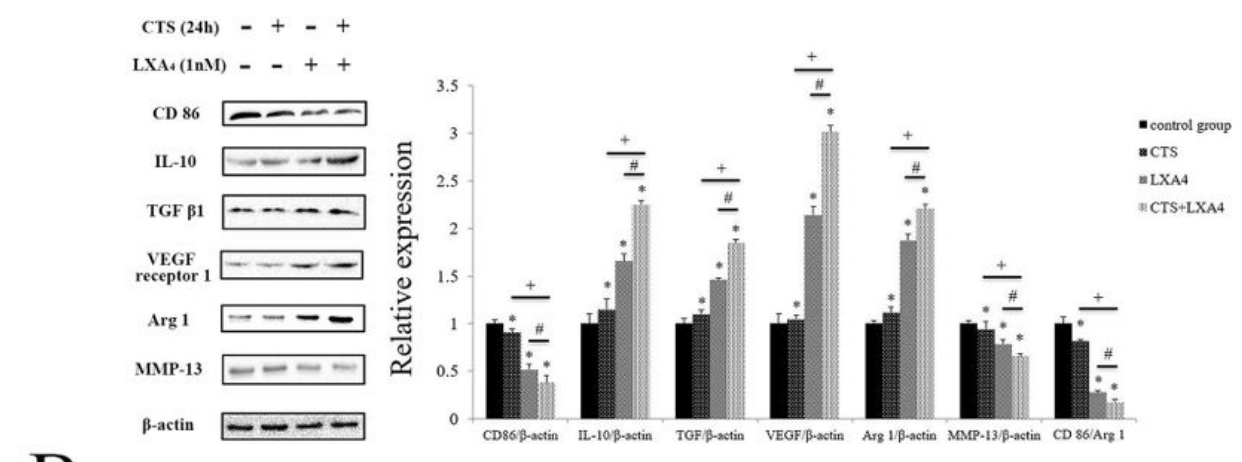

B

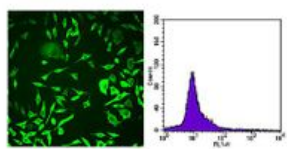

control group

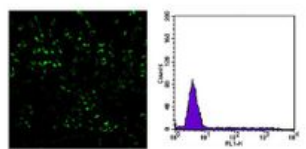

$\mathrm{LXA}_{4}(1 \mathrm{nM})$

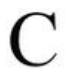

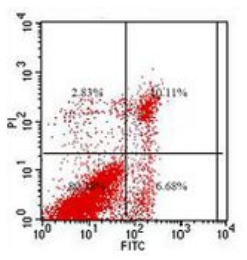

control group

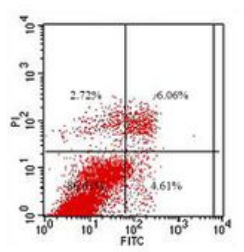

$\mathrm{LXA}_{4}(1 \mathrm{nM})$
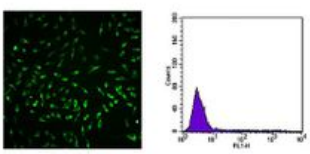

CTS (24h)

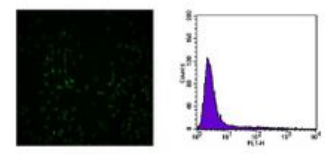

CTS $(24 \mathrm{~h})+\mathrm{LXA}_{4}(1 \mathrm{nM})$

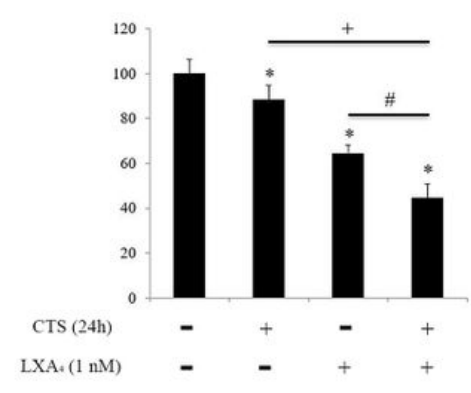

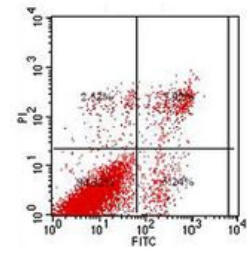

CTS (24h)

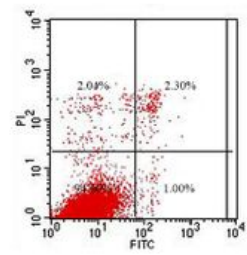

CTS (24h) $+\operatorname{LXA}_{*}(1 \mathrm{nM})$

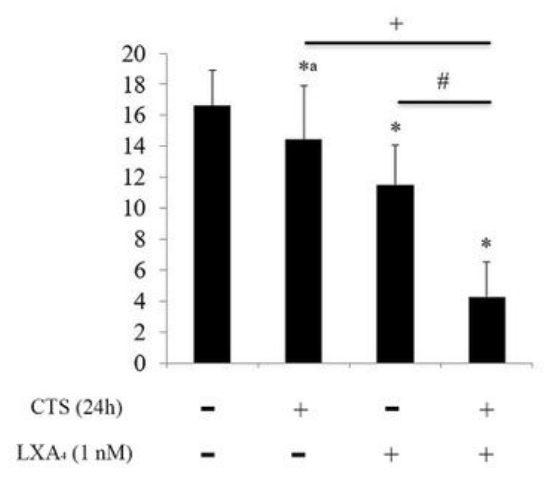

Figure 3

Western blotting, immunofluorescence analysis, and apoptosis in synovial macrophages (A) Western blots for CD86, IL10, TGF- 31 , VEGF receptor 1, Arg 1, and MMP-13. (B) ROS fluorescence microscopy and flow cytometry of synovial macrophages. (C) Synovial macrophage apoptosis analysis by flow cytometry. Differences between the control and treated groups $\left({ }^{\star} P<0.001,{ }^{*} \mathrm{P}=0.040\right)$, CTS-induced synovial macrophages and those subjected to CTS with LXA $4{ }^{+} P$ $<0.001), \mathrm{LXA}_{4}$-induced synovial macrophages and those subjected to CTS with $\operatorname{LXA}_{4}\left({ }^{\#} P<0.001\right)$ were significant (ANOVA). Data are presented as means $\pm 95 \% \mathrm{Cl} ; n=$ three per group. 


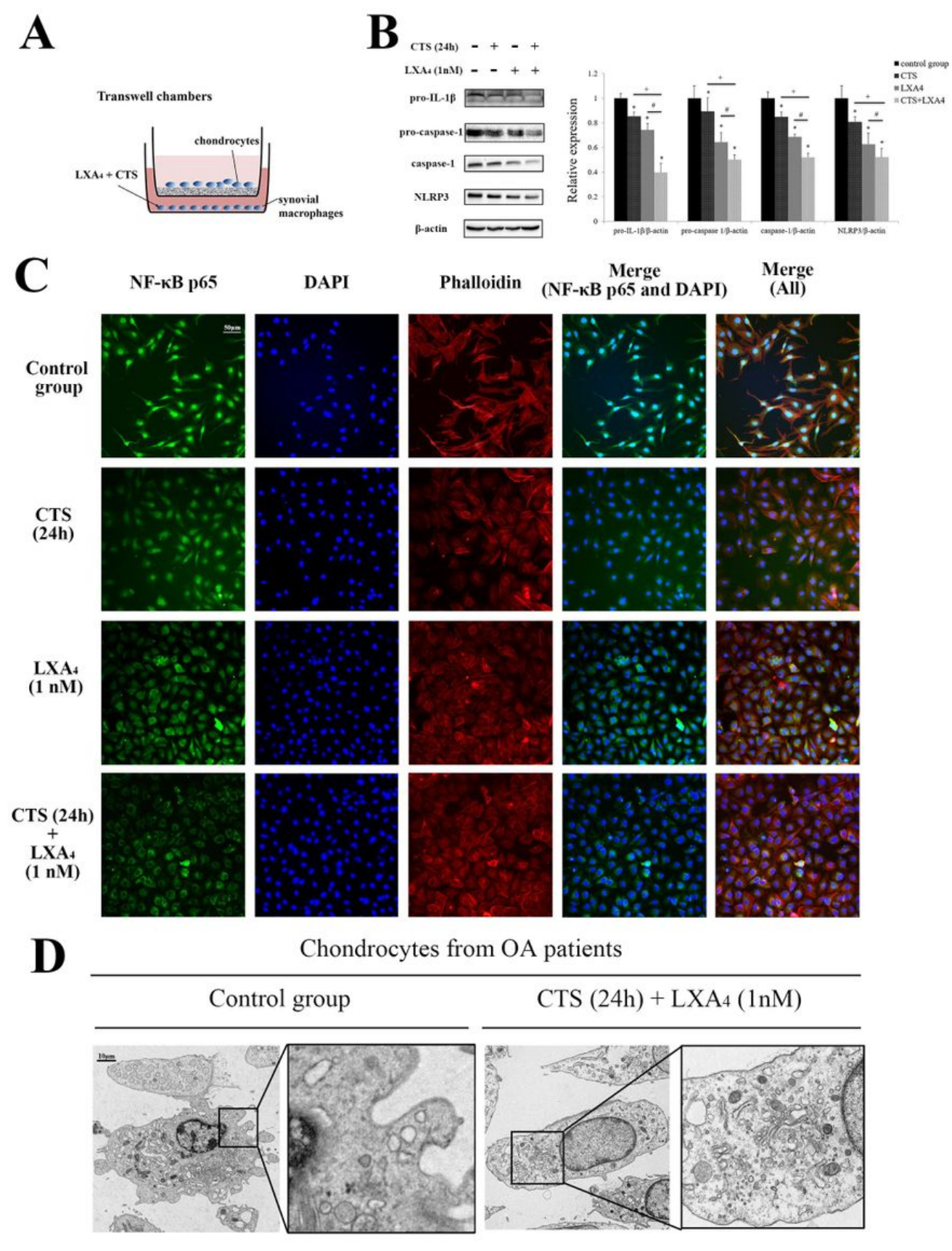

Figure 4

Western blotting, immunofluorescence analysis, and TEM images of chondrocytes (A) Co-culture of synovial macrophages and chondrocytes using transwell chambers. (B) Western blots of pro-IL-1 $\beta$, pro-caspase-1, caspase-1, and NLRP3 in chondrocytes. Differences between the control and treated groups $\left({ }^{\star} P<0.001,{ }^{*} P=0.040\right)$, CTS-induced synovial macrophages and those subjected to CTS with $\mathrm{LXA}_{4}\left({ }^{+} P<0.001\right), \mathrm{LXA}_{4}$-induced synovial macrophages and those subjected to CTS with $\mathrm{LXA}_{4}\left({ }^{\#} P<0.001\right.$ ) were significant (ANOVA). Data are presented as means $\pm 95 \% \mathrm{Cl} ; \mathrm{n}=$ three per group. (C) Effects of CTS and $\mathrm{LXA}_{4}$ on the nuclear translocation of NF-kB p65 in chondrocytes. Cells were immunostained with anti-NF-kB p65 rabbit antibody (green) and visualized by confocal microscopy. Cytoskeleton structures were defined by phalloidin (red) and DAPI (blue) in the nucleus. Scale bar $=50 \mu \mathrm{m}$. (D) TEM images show that $\mathrm{LXA}_{4}(1 \mathrm{nM})+\mathrm{CTS}(24 \mathrm{~h})$ could treat the cellular morphology, including cell swelling, cell membrane protruding and cell nucleus atrophy in chondrocytes from OA patients. 
A

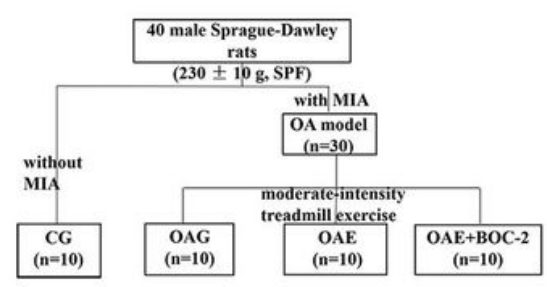

B

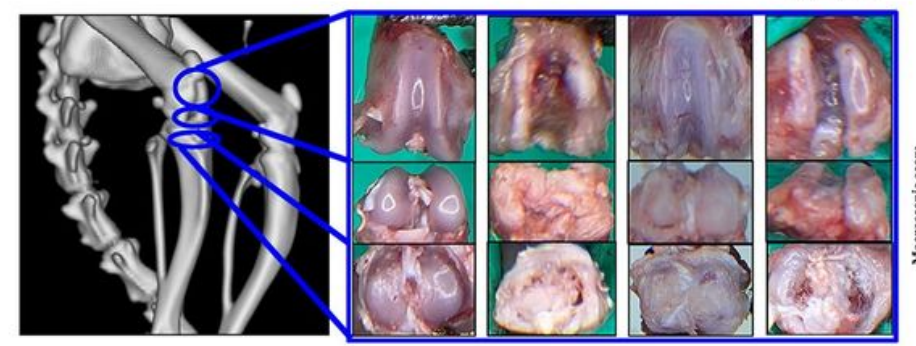

C

$\mathrm{D}$

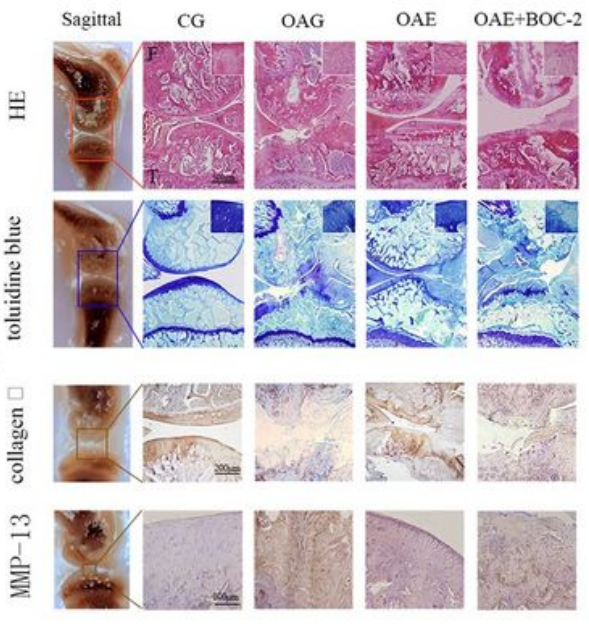

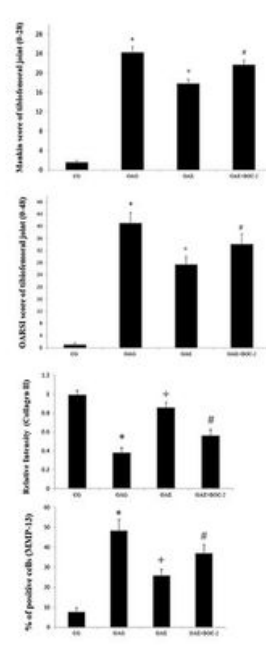

$\mathrm{E}$

CG OAG OAE OAE+

BOC-2

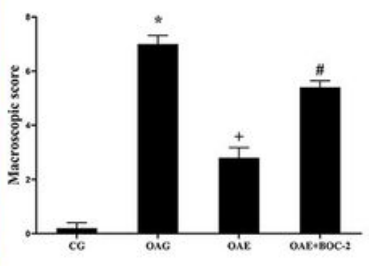

CG OAG OAE OAE+BOC-2

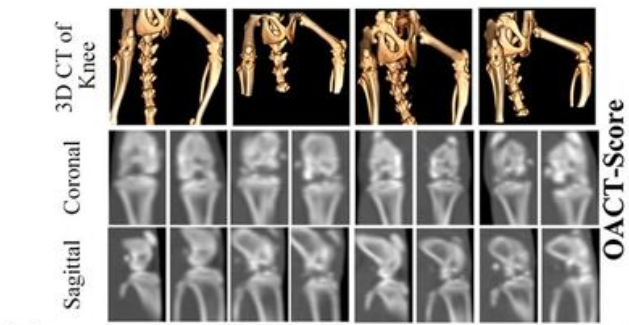

$\mathrm{F}$

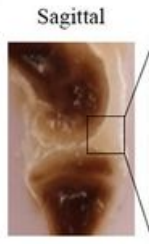

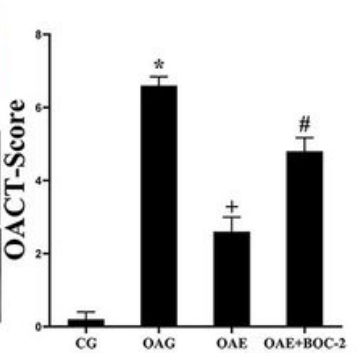
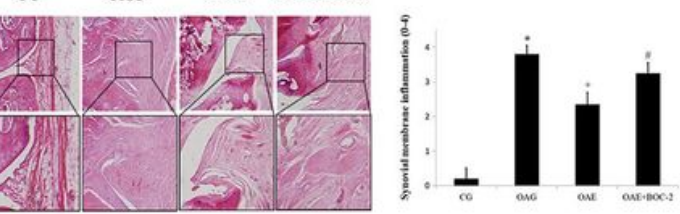

\section{Figure 5}

Histological evaluation of tibiofemoral joints (A) Animal experiment design ( $n=10$ rats/group). (B) Gross imaging of different groups. (C) Histological features of representative tibiofemoral joints sectioned in the sagittal plane, stained with H\&E and toluidine blue. Mankin and OARSI histological scores are shown for each image. F: femur, T: tibia. Mankin scores of tibiofemoral joints. Differences between CG and OAG $\left({ }^{*} P<0.001\right)$, OAG and OAE $\left({ }^{+} P<0.001\right)$, OAE and OAE + BOC-2 ( $\left.{ }^{\#} P<0.001\right)$ were significant. OARSI histological scores for cartilage of tibiofemoral joints. Differences between CG and OAG $\left({ }^{*} P<0.001\right)$, OAG and OAE $\left({ }^{+} P<0.001\right)$, OAE and OAE + BOC-2 $\left({ }^{\#} P<0.001\right)$ were significant. The data are presented as means $\pm 95 \% \mathrm{Cl} ; n=10$ rats per group. (D) Micrographs show the relative intensities of collagen II staining and the percentages of MMP13-positive cells in articular cartilage of each experimental group. (E) Computed tomography indicated that joint space narrowing, osteophytosis, and subchondral cysts were heavier in OAG and OAE + BOC-2, but OAE relieve these symptoms. (F) Histological features of synovium and microscopic score to evaluate the synovial membrane inflammation levels. Differences between CG and OAG $\left({ }^{*} P<0.001\right)$, OAG and OAE $\left({ }^{+} P<0.001\right)$, and $\mathrm{OAE}$ and OAE + BOC-2 ( $\left.{ }^{*} P<0.001\right)$ were significant (ANOVA). Data are presented as means $\pm 95 \% \mathrm{Cl} ; \mathrm{n}=$ five rats per group. Experimental groups: $C G=$ control group; $O A G=O A$ group injected with $M I A ; O A E=O A G$ subjected to moderateintensity treadmill exercises; $\mathrm{OAE}+\mathrm{BOC}-2=\mathrm{OAE}$ conditions plus $\mathrm{BOC}-2$. 
A

B
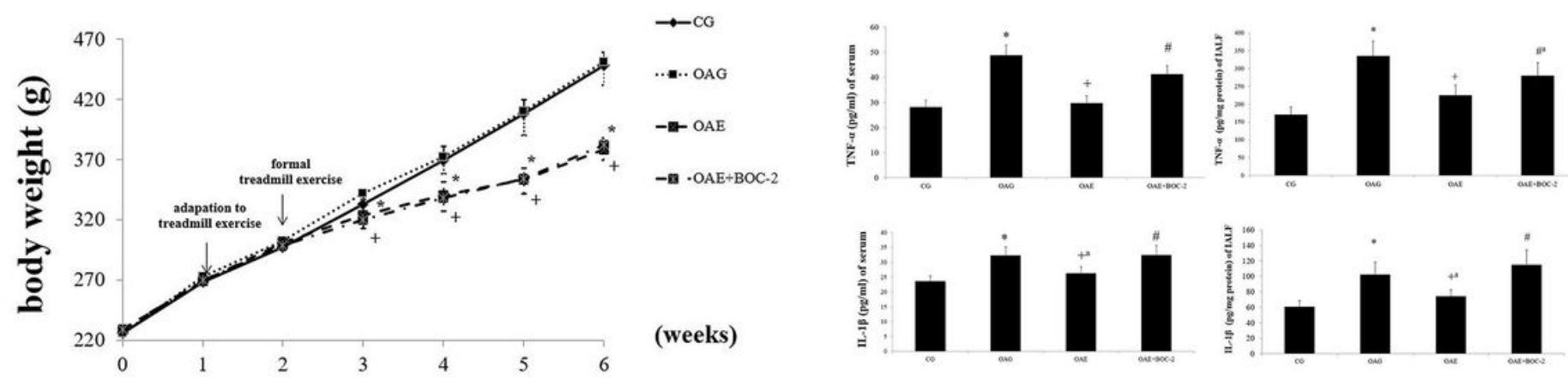

$\mathrm{C}$
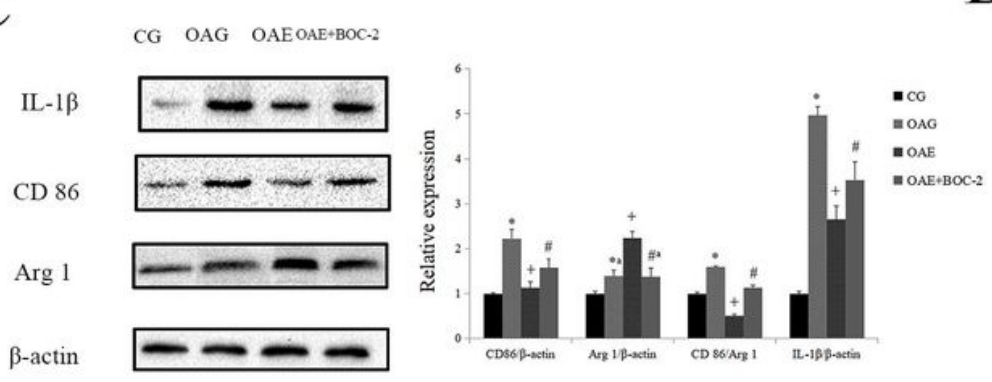

$\mathrm{D}$

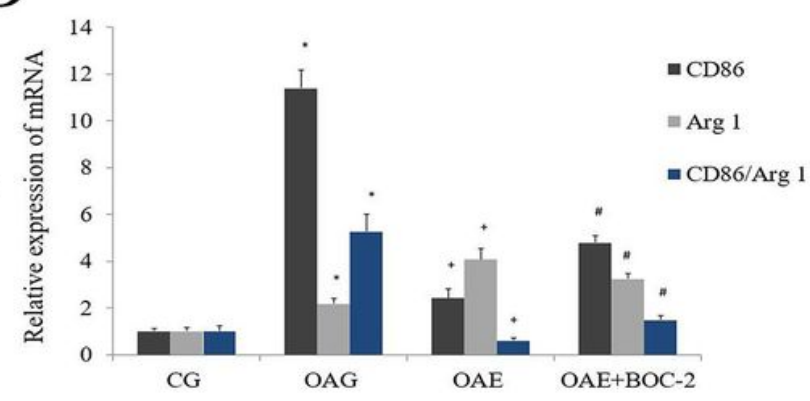

\section{Figure 6}

Body weight, ELISA, western blot analyses, and qPCR (A) Body weight comparisons. No significant differences were observed between rat body weight in the $C G$ and $O A G$ groups. Differences in body weight between OAG and OAE ( $P<$ 0.001), and OAG and OAE + BOC-2 $\left({ }^{+} P<0.001\right)$ were significant (ANOVA). Data are presented as means $\pm 95 \% \mathrm{Cl} ; \mathrm{n}=10$ rats per group. (B) IL-1 $\beta$ and TNF-a levels in serum and IALF. Differences between CG and OAG $\left({ }^{*} P<0.001,{ }^{\text {*a }} P=0.001\right)$, OAG and OAE $\left({ }^{+} P<0.001,{ }^{\text {+a }} P=0.002\right)$, and OAE and OAE + BOC-2 $\left({ }^{\#} P<0.001,{ }^{\# a} P=0.012\right)$ were significant (ANOVA). Data are presented as means $\pm 95 \% \mathrm{Cl} ; n=10$ rats per group. (C) Protein levels were determined in synovial tissue by western blotting. Differences between CG and OAG $\left({ }^{\star} P<0.001,{ }^{*} \mathrm{a}=0.006\right), \mathrm{OAG}$ and OAE $\left({ }^{+} P<0.001\right)$, and OAE and OAE + BOC-2 ( $\left.{ }^{\#} P<0.001,{ }^{\# a} P=0.001\right)$ were significant (ANOVA). $\beta$-actin was used as an internal control. Data are presented as means $\pm 95 \% \mathrm{Cl} ; \mathrm{n}=$ three rats per group. (D) CD86 and Arg1 mRNA expression in synovium in different group was determined by qPCR. Differences between CG and OAG $\left({ }^{\star} P<0.001\right)$, OAG and OAE $\left({ }^{+} P<0.001\right)$, and OAE and OAE + BOC-2 ( $\left.{ }^{\#} P<0.001\right)$ were significant (ANOVA). Data are presented as means $\pm 95 \% \mathrm{Cl} ; \mathrm{n}=$ nine per group. Experimental groups: $C G=$ control group; $O A G=O A$ group induced by MIA; OAE = OAG subjected to moderate-intensity treadmill exercise, and $\mathrm{OAE}+\mathrm{BOC}-2$, $\mathrm{OAE}$ with $\mathrm{BOC}-2$ administered by intraperitoneal injection. 


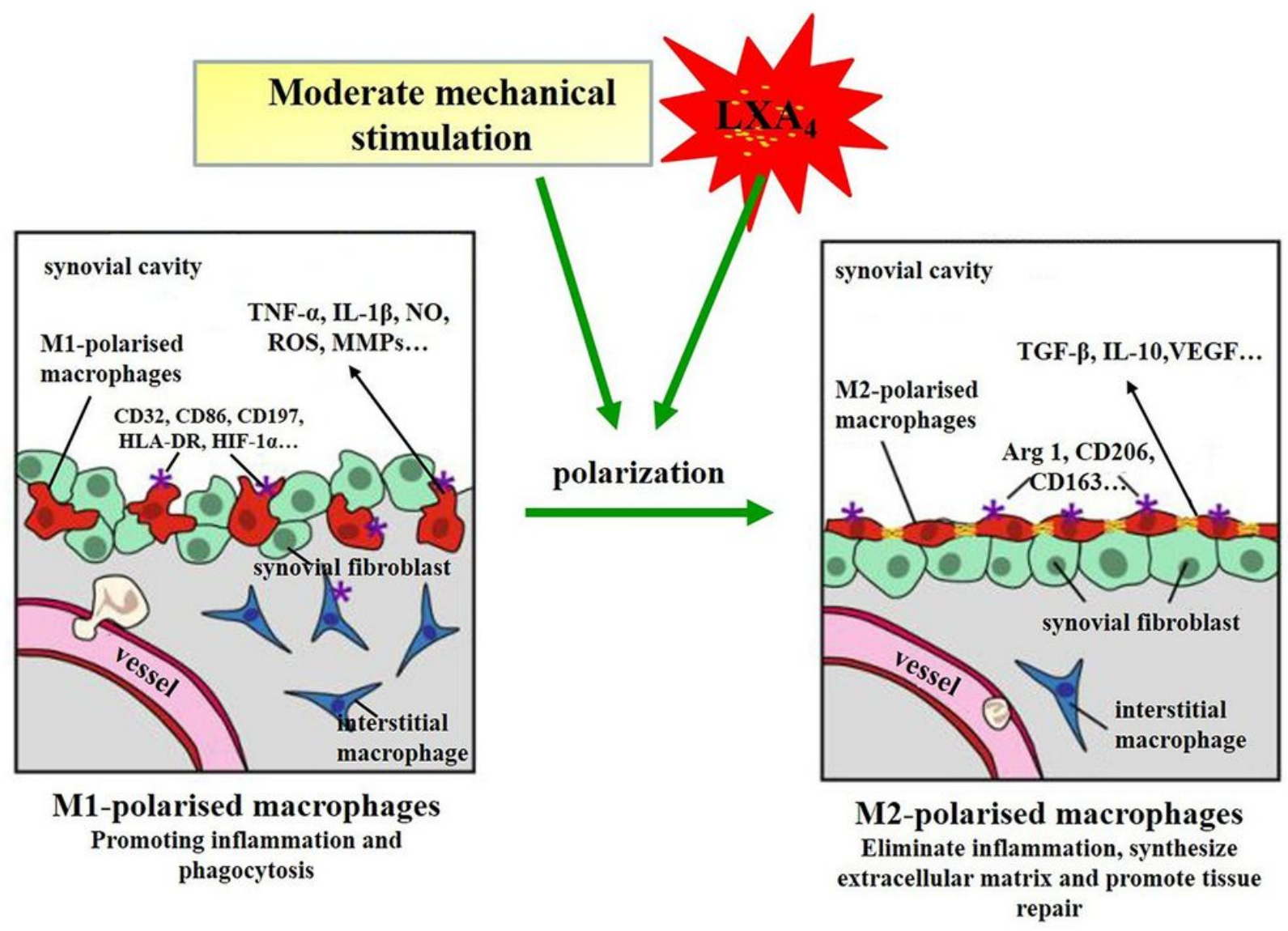

Figure 7

Mechanical stress protects against chondrocyte pyroptosis through $L X A_{4}$ via synovial macrophage $M 2$ subtype polarization. Mechanistic hypothesis of $\mathrm{LXA}_{4}$ involvement in OA exercise therapy. Moderate mechanical stimulation and $\mathrm{LXA}_{4}$ facilitate $\mathrm{M} 2$ polarization of synovial macrophages.

\section{Supplementary Files}

This is a list of supplementary files associated with this preprint. Click to download.

- Supplementaryfigure1.jpg 